\title{
Citation:
}

Salvador, C.E., Berg, M.K., Yu, Q., San Martin, A. \& Kitayama, S. (in press) Relational mobility predicts a faster spread of COVID-19: A 39 country study, Psychological Science.

\section{Relational mobility predicts a faster spread of COVID-19: \\ A 39 country study}

\author{
Cristina E. Salvador ${ }^{1}$ \\ Martha K. Berg ${ }^{1}$ \\ Qinggang $\mathrm{Yu}^{1}$ \\ Alvaro San Martin² \\ Shinobu Kitayama ${ }^{1}$
}

${ }^{1}$ University of Michigan, ${ }^{2}$ IESE Business School

Author Contributions: CES and SK drafted the paper; CES, MKB, and QY carried out statistical analysis; MKB, QY, and ASM provided critical revisions on the draft.

Address correspondence to Cristina Salvador or Shinobu Kitayama at Department of Psychology, University of Michigan, Ann Arbor, MI 48109 USA. Emails: csalvad@umich.edu and kitayama@umich.edu. 


\begin{abstract}
It has become increasingly clear that COVID-19 transmits between individuals. It stands to reason that the spread of the virus depends on sociocultural ecologies that facilitate or inhibit social contact. In particular, the community-level tendency to engage with strangers and freely choose friends, called relational mobility (RM), entails increased opportunities to interact with a larger and more variable range of others. It may therefore be associated with a faster spread of infectious diseases, including COVID-19. Here, we tested this possibility by analyzing growth curves of confirmed cases and deaths of COVID-19 in the first 30 days of the outbreaks in 39 countries. We found the growth was significantly accelerated as a function of a country-wise measure of RM. This relationship was robust either with or without a set of control variables, including demographic variables, reporting bias, testing availability, and cultural dimensions of individualism and government efficiency. Policy implications are discussed.
\end{abstract}




\section{Statement of Relevance}

The COVID-19 pandemic has impacted all countries on the globe. However, some countries have suffered far more than some others. It is of utmost significance to understand factors explaining this cross-country variability. Here, we report the first evidence that the variation in vulnerability to COVID-19 may be due in part to cultural practices of social relationships. In particular, we found the spread of this virus depends on how broad or narrow the range of people one relates to and interacts with -- a dimension called relational mobility (RM).

Compared to their low RM counterparts, countries higher in RM showed a significantly steeper slope, indicating a faster spread of the virus during the early period of country-wise outbreaks. This evidence underscores the need for social distancing to "flatten the curve," especially in countries that value social openness. 


\section{Introduction}

Over the last several months, a novel strain of coronavirus (SARS-CoV-2) has spread across the globe causing the COVID-19 pandemic. While there is substantial cross-national variation in the damage incurred by the virus, little is known about the factors that contribute to this variation. It is commonly accepted, however, that the virus transmits through social contact. Hence, viral transmission could increase as social contact becomes more frequent and variable. We thus investigated whether country-wise vulnerability to COVID-19 might vary systematically based on social ecologies that encourage or discourage social contact.

Our focus is on relational mobility (RM), the extent to which it is easy to form new relationships and terminate current ones in any given society (Yuki \& Schug, 2020). In societies low in RM, interdependence with close others is valued (Markus \& Kitayama, 1991).

Relationships are typically ascribed by social roles and restricted to close others (Adams, 2005). Conversely, in societies high in RM, social relationships tend to be freely chosen, and more expansive. People can form new relationships and leave former ones at will. They thus tend to be socially open (Schug et al., 2010). The resulting social ecologies would increase the opportunity for interaction with a greater number of individuals outside each person's primary social groups (e.g., close inner circle of friends). Thus, high RM may put people at particularly high risk for contracting an infectious disease like COVID-19.

We tested whether country-wise RM scores (Thomson et al., 2018) would positively predict the growth of cases and deaths due to COVID-19. The growth (the rate of increase) is unlikely to be affected by reporting biases and testing availability, as long as the latter factors are constant. It would therefore be important to examine a short initial period of growth. At the same time, it is necessary to test a sufficiently long period to obtain reliable estimates of the growth rate. To simultaneously meet these two competing demands, we analyzed the first 30 days of country-wise outbreaks in the main analysis. This analytic strategy also enabled us to capture the COVID-19 spread prior to lockdowns. 


\section{Methods}

\section{Data}

Main variables. We retrieved data on daily confirmed COVID-19 cases and deaths by country from a public repository updated daily by the Johns Hopkins University Center for Systems Science and Engineering (https://github.com/CSSEGISandData/COVID-19). Our current results are based on data up until July 21, 2020. RM scores were obtained from Thomson et al. (2018), who measured RM by assessing the extent to which people perceive others in their local communities are socially open and, thus, seek new friendships while exiting unsatisfactory relationships. They administered a 12-item scale of RM to a large number of adults, recruited with Facebook ads, in 39 countries, and found systematic cross-cultural variation. A series of analyses with a wide range of culture-level indicators of behavioral outcomes shows the validity of the RM score. For example, RM predicts the national levels of general trust, self-disclosure, intimacy, and social support (Thomson et al., 2018).

We followed prior work (Berg et al., 2020), and included countries if they had reported at least 15 days of data, starting with the day of the first 100 confirmed cases as 'day 1'. For deaths, we included countries with at least 15 days of data, starting with the first day of at least 1 reported death as 'day 1.' All 39 countries met this criterion.

The 39 countries included are listed in Table S1, which also shows the dates for the first 100 confirmed cases, the first confirmed death, and the date of national lockdown if it was instituted, for each of the countries. In many cases, the lockdown occurred during this period. Even in those cases, it occurred more than halfway through the period. Since it takes a certain amount of time (usually several weeks, based on an estimate for the 1918 influenza pandemic by Bootsma \& Ferguson, 2007) for any lockdown to have an effect, it would seem reasonable to ignore any effect of state-imposed lockdowns on the current analysis. This point is arguably valid in a robustness check with the first 15 days of data. 
Demographics. Following prior work (Berg et al., 2020), several demographic variables were included as covariates. Total population was added because larger groups of people will have more cases and deaths. Median age of the total population (in years) was included since older adults are more susceptible to disease. Population density (in persons per square kilometer) was used because it is likely to foster greater social contact, resulting in greater chances of infection. Net migration (persons entering country minus persons exiting country, per 1000 people) was included so as to control for population movement. These four statistics were compiled from the United Nations Department of Economic and Social Affairs World Population Prospects 2019 (United Nations, Department of Economic and Social Affairs, Population Division, 2018). Gross domestic product (at purchasing power parity) per capita (GDP per capita in thousands), tourism rates and percent urban were included to control for economic development, the influx of foreigners and how urban the country is (The World Bank, 2019).

Cultural dimensions. Three cultural dimensions were tested as potential confounding variables. First, Hofstede's index of individualism (Hofstede, 1984) was used because its conceptual equivalent (independent self-construal) is positively correlated with RM (San Martin et al., 2019; Thomson et al., 2018), consistent with the notion that freedom to choose is an important facet of independent self-construal (Markus \& Kitayama, 1991). Scores are based on responses to a series of questions asked to employees of a large IT company across countries. Scores were available for 35 of the 39 countries. Second, the efficiency in governmental operations might promote more effective coping with COVID-19. Hence, we controlled for this variable as in recent work (Gelfand et al., 2020). We used the World Bank's Government Efficiency Index -- an index that shows the public sector's performance in managing and regulating the political economy (retrievable at: https://bit.ly/34IXAT9). The index varies from 1 (very inefficient) to 5 (highly efficient). Scores were available for 34 of the 39 countries. Third, recent research suggests that the tightness (vs. looseness) of social norms could be an adaptation to threats, including pathogen threats (Gelfand et al., 2011). Tightness might then 
lower the growth rate of cases and deaths. The index, adopted from Gelfand et al. (2020), is an arithmetic mean of responses to a 6-item questionnaire assessing the perceived rigidity of social norms in one's own country. Scores were available for 24 of the 39 countries tested.

BCG. Berg et al. (2000) tested 139 countries and found that those with universal Bacillus Calmette-Guérin (BCG) vaccination policies show a reduced rate of the growth of both COVIDrelated confirmed cases and deaths (Berg et al., 2020). We thus used BCG policy status (with or without BCG policy) as another covariate. Data was available for 37 out of the 39 countries.

\section{Robustness Checks}

Underreporting of cases. Countries may vary in underreporting due to, for example, governmental information suppression. To account for this, we adopted an underreporting index devised by Russell and colleagues (Russell et al., 2020). These researchers first computed a case fatality ratio (CFR) in each country that is adjusted for delay between admission to the hospital and death. Then they computed the ratio of the best empirical estimate of CFR (1.4\%) to the adjusted CFR for each country. If this ratio is smaller than one, it indicates underreporting of cases. Some countries, such as Italy, Spain, and Morocco, show substantial underreporting (underreporting index $<10 \%$ ), whereas some others, such as Norway, Israel, and South Korea, show very low underreporting (underreporting index $>50 \%$ ). We used country-wise underreporting scores on April 15, 2020, downloaded from https://github.com/thimotei/CFR_calculation. This index was available for 29 of the 39 countries.

This index can be defined as a measure of inaccuracy of the report of cases. In one analysis, we used it as a weight, with the data from countries with higher values of this index weighed more than the data from those with lower values. The index can also indicate underestimation of the number of cases reported. Thus, in another analysis, we also used it as an additional covariate. These analyses were performed only for the number of confirmed cases.

Testing availability. We also adopted the number of COVID-19 tests per case. This data was obtained from: https://ourworldindata.org/grapher/the-number-of-tests-for-each- 
confirmed-case-since-5th-death. In countries such as France and Mexico, the tests per case ratio was low, suggesting that testing was not readily available. Conversely, in countries like New Zealand, Australia and Taiwan, this ratio was high, implying higher degrees of testing availability. Data was available for 29 out of the 39 countries. As with the reporting index, we conducted two analyses with these scores. First, we weighed countries with more testing more heavily presumably because more testing would lead to more accurate counts of cases.

Second, we included it as a covariate since less testing can also lead to an underestimation of cases reported. These analyses were performed only for the number of confirmed cases.

$15 / 60$ days of country-wise outbreaks. In the main analyses, we focused on the initial 30-day period of country-wise outbreaks. To ensure the robustness of the pattern, we carried out two analyses that used a half (15 days) or twice as many days (60 days) as in our standard analysis.

Interpolation of RM data with cultural distance scores. In a recent study, Muthukrishna and colleagues (2020) offered an index of cultural distance between each of the pairs of 80 countries. The distance index is based on geometric distance across many attitudinal items culled from the World Value Survey. The 80 countries tested by Muthukrishna et al. (2020) included 34 of the 39 countries for which RM scores were available. By averaging the RM scores for the 34 countries after weighting each RM score with the inverse of the cultural distance score of each of the countries, we interpolated the RM score for each of the 46 (= 8034) countries. For example, consider one of the 80 countries tested by Muthukrishna et al. (2020) that did not have an RM score (designated as $R M_{j}$ ). This country has a cultural distance score (designated as $D_{i j}$, where $\mathrm{i}=1$ to $\mathrm{M}$ ) with each of the $\mathrm{M}$ countries with RM scores (designated as $R M_{i}$ ). The RM score for this country was computed by the following formula: 


$$
R M_{j}=\frac{\sum_{i=1}^{m} R M_{i} * \frac{1}{D_{i j}}}{\sum_{i=1}^{m} \frac{1}{D_{i j}}}
$$

In this instance, $M=34$. The RM scores imputed for the 46 countries were used to repeat the same set of analyses for the $85(=39+46)$ countries.

To assess the validity of this procedure, we repeated the same procedure for the 34 countries for which the RM scores were available. In this case, to interpolated the RM score for a target country by averaging the RM scores for the remaining 33 countries (i.e., all countries for which RM scores were available minus the target country) after weighting each score with the inverse of the cultural distance between the target country and each of the 33 countries. In this instance, $M=33$. The correlation between actual RM scores and the interpolated RM scores was .596, $p<.001$.

\section{Statistical Analysis}

All analyses were conducted on up to 30 days of data from each country. Linear mixed effect models with restricted maximum likelihood estimation were used to analyze both the number of cases and deaths. We natural log-transformed both cases and deaths since the growth of cases and deaths is known to be exponential in an early period of a pandemic. Day was centered so that main effects could be interpreted as differences at the mean day of the growth curve. Total population was natural log-transformed to reduce skewness. All demographic and cultural variables were z-scored. We estimated both a random intercept and random slope across days to allow for the heterogeneity of growth curves across countries. We included another random effect that accounts for countries being nested in Geographic regions defined by the World Bank (The World Bank, 2019), because these nations are not independent and have some shared cultural and political history.

We tested three models. Model 1 included Day, RM, the Day x RM interaction. To control for population size, both Population size and its interaction with Day were also included. Model 2 added all the demographic variables to Model 1. All demographic variables were 
available for all 39 countries, with the exception of the Tourism measure for Taiwan. Model 3 included only those covariates that had an interaction with Day at $p<.10$ in Model 2. We report the statistics from Model 3 in the text. All other statistics can be found in relevant tables. Regarding the remaining covariates (e.g., cultural values, BCG policy status, underreporting, and testing availability), data were often missing for some countries. Thus, to retain the maximal number of countries, they were tested one at a time in a separate analysis. When a full model failed to converge, we dropped the intercept-slope covariance of the random effects (Country and Geographic region). This is arguably the most conservative strategy, as the covariance terms would be automatically dropped once one of the two terms defining the covariances was dropped (Bates et al., 2018). When the model still did not converge, we dropped the Geographic region as a nesting factor although this happened only in for a few robustness checks.

Correlations among the cultural and demographic variables are given in Figure S1.

\section{Results}

\section{Main Analysis}

Confirmed cases. Results are summarized in Table 1-A. The main effect of Day was significant, $b=.121, p=.006$, showing an increase in COVID-19 cases over time. Importantly, it was qualified by a significant Day by RM interaction, $b=.112, p=.002$. Fig.1-A shows the growth of confirmed cases in the natural log scale. Countries higher in RM showed a faster growth of confirmed cases over time compared to countries low in RM. The main effect of RM was also significant, $b=1.80, p=.010$. This main effect, however, is a necessary consequence of the steeper slope in the high (vs. low) RM countries. Hence, in and by itself, it does not carry any theoretical significance. The beta coefficients indicating the growth rate are plotted in Fig. 1$B$, which shows that the relationship between the growth rate and RM is robust and unlikely to be due to any outliers. Among the demographic variables, total population (designated as Population), migration and tourism had an impact on the growth rate. Countries with larger populations had a faster rate of the growth of confirmed cases, as indicated by the Day $\mathrm{x}$ 
Population interaction, $b=.021, p=.003$. Moreover, countries with more migration and tourism had a faster rate of the growth of confirmed cases, as indicated by the Day $\mathrm{x}$ Migration and Day $\mathrm{x}$ Tourism interaction, $b=.014, p=.017$ and $b=.024, p=.001$, respectively. The remaining covariates had no significant effect on the growth rate of confirmed cases.

RM accounted for a sizable amount of variance in the number of confirmed cases. We hypothesize that RM fosters a higher rate of the increase by Day (as captured by the Day x RM interaction) and, by so doing, increases the number of cases by the end of the study period. Hence, the total amount of variance explained by RM is the sum of the variance explained by the Day $x$ RM interaction and the RM main effect (obtained by subtracting the variance explained by a model including Day only from a model including Day, RM, and Day $x$ RM). This combined variance was $8.4 \%$.

Deaths. Results are summarized in Table 1-B. The main effect of Day was significant, $b$ $=.149, p=.002$, showing an increase in the number of deaths across time. The Day $\mathrm{x}$ RM interaction was also significant, $b=.144, p=.010$. Deaths increased over time, and were greater for countries high in RM (Figure 1-C). This effect was statistically significant in both Models 1 and 3 although it was marginal in Model 2. As shown in Figure 1-D, it is not due to any outliers. As in the analysis of confirmed cases, the Day x Population interaction was significant, $b=.031, p=.004$, showing that countries with larger populations had a higher growth rate of deaths. Other demographic variables had no effect. RM accounted for $7.5 \%$ of the variance in the number of deaths.

\section{Controlling for Other Cultural Variables}

Individualism. In an analysis performed on 35 of the 39 countries, after controlling for individualism, we found a significant Day $\times$ RM interaction for cases, $b=.084, p=.010$ (Table S2-A). This interaction was marginal for deaths, $b=.101, p=.063$ (Table S2-B).

Government efficiency. In an analysis performed on 34 of the 39 countries, after controlling for government efficiency, the Day $x$ RM interaction remained significant for both 
cases and deaths respectively, $b=.116, p=.001$ and, $b=.115, p=.032$ (see Tables S3-A and B).

Tightness. In an analysis performed on 24 of the 39 countries, after controlling for Tightness, the Day x RM interaction became marginal for cases and non-significant for deaths, $b=.087, p=.065$ and $b=.071, p=.119$, respectively (Table S4-A and B). The weaker Day $\mathrm{x}$ RM interaction may be due to diminished sample size (= 24).

BCG. In an analysis performed on 37 of the 39 countries, after controlling for BCG policy status, the Day x RM interaction was significant for cases, $b=.088, p=.004$ (Table S5-A). This effect was no longer significant for deaths, $b=.083, p=.160$ (Table S5-B).

\section{Robustness Checks}

Underreporting of cases. In an analysis performed on confirmed cases on 29 out of the 39 countries, with the Russell et al. (2020) underreporting index as a weighting factor, the Day $x$ RM interaction remained significant, $b=.095, p=.004$ (Table S6-A). When the underreporting index was used as an additional covariate, the Day $\times$ RM interaction remained significant, $b=$ $.080, p=.025$ (Table S6-B)

Testing availability. In an analysis performed on cases for 29 out of the 39 countries, with the test availability index as a weighting factor, the Day $x$ RM interaction remained significant, $b=.085, p=.015$ (Table S7-A). When it was used as an additional covariate, the Day x RM interaction remained significant, $b=.076, p=.026$ (Table S7-B).

The first 20 cases. In an analysis performed on 38 out of the 39 countries, with the 100 (rather than 20) cases as the cutoff, the Day $\times$ RM interaction remained significant for cases, $b=$ $.131, p=.010$ (Table S8).

The first-15 days of country-wise outbreaks. When the data from the first 15 (rather than 30) days were analyzed, the Day $x$ RM interaction remained significant for cases, $b=.118$, $p=.029$ (Table S9-A). This interaction was no longer significant for deaths, $b=.134, p=.172$, plausibly due to the reduced amount (one half) of data included in this analysis (Table S9-B). 
The first-60 days of country-wise outbreaks. When the data from the first 60 (rather than 30) days were analyzed, the Day $x$ RM interaction remained significant for cases, $b=.039$, $p=.038$ (Table S10-A) and deaths, $b=.086, p=.001$ (Table S10-B).

Interpolation of RM data with cultural distance scores. Lastly, we examined whether the same results were present if we increased the number of countries from 39 to 85 by using the interpolated RM scores for 46 countries for which the RM scores were unavailable. As shown in Table S11, the Day $\times$ RM interaction was significant for both cases and deaths, $b=$ $.090, p=.007$ and $b=.113, p=.022$, respectively.

\section{Discussion}

Our findings show that each country's social openness (called relational mobility, RM) positively predicts the growth rate of both confirmed cases and deaths of COVID-19 during an early period of country-wise outbreaks. The results for cases were robust across a number of analyses that controlled for underreporting, testing availability, demographic variables, and cultural traits such as individualism and government efficiency. Although a comparable effect for deaths was less robust when the inclusion of covariates necessitated a reduction of the number of countries that could be included, it was significant in the main analysis that included all the 39 countries. The RM effect was sizable. RM accounted for approximately $8 \%$ of variance for both cases and deaths. To illustrate, the U.S. is among the highest in RM. If it had been low in RM, comparable to Japan (one of the lowest in RM), the deaths at the end of the 30-day study period would have been $8.2 \%$ (281) of the actual number reported (3417).

The COVID-19 pandemic has proven extremely difficult to contain. Without any vaccines available, the only viable defense against the virus is to keep sufficient physical distance from others, particularly, strangers. Our data suggests that this practice of social distancing could prove indispensable in countries high in RM. In such countries, individuals might seek new friends and acquaintances outside of their primary groups (Thomson et al., 2018); they might be more outgoing (Kim et al., 2018); and they might not easily suppress emotions in face-to-face 
encounters (Kraus \& Kitayama, 2019). These psychological propensities could make social ecologies particularly vulnerable to infectious disease.

Some limitations must be noted. First, we focused only on an early period of outbreaks to minimize various confounds. Second, country is admittedly a crude unit of analysis. Third, our sample size was limited by the availability of RM scores. Nevertheless, our data is the first to show a substantial effect of socio-cultural ecologies on the peril of infectious disease.

In closing, since RM is an important expression of the values of independence, egalitarianism, and freedom of movement and choice (Inglehart \& Baker, 2000; Markus \& Kitayama, 1991), the present findings may be posing a fundamental challenge to all countries aspiring to promote these values. Expertise of social and behavioral sciences (Van Bavel et al., 2020) may therefore be strongly called for to devise strategies to fight against infectious disease without compromising the core values of democracy. 


\section{References}

Adams, G. (2005). The Cultural Grounding of Personal Relationship: Enemyship in North American and West African Worlds. Journal of Personality and Social Psychology, 88(6), 948-968. https://doi.org/10.1037/0022-3514.88.6.948

Bates, D., Kliegl, R., Vasishth, S., \& Baayen, H. (2018). Parsimonious Mixed Models. ArXiv:1506.04967 [Stat]. http://arxiv.org/abs/1506.04967

Bootsma, M. C. J., \& Ferguson, N. M. (2007). The effect of public health measures on the 1918 influenza pandemic in U.S. cities. Proceedings of the National Academy of Sciences, 104(18), 7588-7593. https://doi.org/10.1073/pnas.0611071104

Berg, M. K., Yu, Q., Salvador, C. E., Melani, I., \& Kitayama, S. (2020). Mandated Bacillus Calmette-Guérin (BCG) vaccination predicts flattened curves for the spread of COVID19. Science Advances, eabc1463. https://doi.org/10.1126/sciadv.abc1463

Gelfand, M. J., Jackson, J. C., Pan, X., Nau, D., Dagher, M. M., \& Chiu, C. (2020). Cultural and Institutional Factors Predicting the Infection Rate and Mortality Likelihood of the COVID19 Pandemic [Preprint]. PsyArXiv. https://doi.org/10.31234/osf.io/m7f8a

Gelfand, M. J., Raver, J. L., Nishii, L., Leslie, L. M., Lun, J., Lim, B. C., Duan, L., Almaliach, A., Ang, S., Arnadottir, J., Aycan, Z., Boehnke, K., Boski, P., Cabecinhas, R., Chan, D., Chhokar, J., D’Amato, A., Ferrer, M., Fischlmayr, I. C., ... Yamaguchi, S. (2011). Differences Between Tight and Loose Cultures: A 33-Nation Study. Science, 332(6033), 1100-1104. https://doi.org/10.1126/science.1197754

Hofstede, G. (1984). Culture's Consequences: International Differences in Work-Related Values (Abridged edition). SAGE Publications, Inc.

Inglehart, R., \& Baker, W. E. (2000). Modernization, Cultural Change, and the Persistence of Traditional Values. American Sociological Review, 65(1), 19. https://doi.org/10.2307/2657288

Kim, H., Schimmack, U., Oishi, S., \& Tsutsui, Y. (2018). Extraversion and life satisfaction: A 
cross-cultural examination of student and nationally representative samples. Journal of Personality, 86(4), 604-618. https://doi.org/10.1111/jopy.12339

Kraus, B., \& Kitayama, S. (2019). Interdependent self-construal predicts emotion suppression in Asian Americans: An electro-cortical investigation. Biological Psychology, 146, 107733. https://doi.org/10.1016/j.biopsycho.2019.107733

Markus, H. R., \& Kitayama, S. (1991). Culture and the self: Implications for cognition, emotion, and motivation. Psychological Review, 98(2), 224-253. https://doi.org/10.1037/0033295X.98.2.224

Muthukrishna, M., Bell, A. V., Henrich, J., Curtin, C. M., Gedranovich, A., Mclnerney, J., \& Thue, B. (2020). Beyond Western, Educated, Industrial, Rich, and Democratic (WEIRD) Psychology: Measuring and Mapping Scales of Cultural and Psychological Distance. Psychological Science, 095679762091678. https://doi.org/10.1177/0956797620916782

Russell, T. W., Hellewell, J., Abbott, S., Golding, N., Gibbs, H., Jarvis, C. I., van Zandvoort, K., Flasche, S., Eggo, R., Edmunds, W. J., \& Kucharski, A. J. (2020). Using a delayadjusted case fatality ratio to estimate under-reporting. CMMID Repository. https://cmmid.github.io/topics/covid19/severity/global_cfr_estimates.html

San Martin, A., Schug, J., \& Maddux, W. (2019). Relational Mobility and Cultural Differences in Analytic and Holistic Thinking. Journal of Personality and Social Psychology, 24.

Schug, J., Yuki, M., \& Maddux, W. (2010). Relational Mobility Explains Between- and WithinCulture Differences in Self-Disclosure to Close Friends. Psychological Science, 21(10), 1471-1478. https://doi.org/10.1177/0956797610382786

The World Bank. (2019). International Comparison Program Database.

Thomson, R., Yuki, M., Talhelm, T., Schug, J., Kito, M., Ayanian, A. H., Becker, J. C., Becker, M., Chiu, C., Choi, H.-S., Ferreira, C. M., Fülöp, M., Gul, P., Houghton-Illera, A. M., Joasoo, M., Jong, J., Kavanagh, C. M., Khutkyy, D., Manzi, C., ... Visserman, M. L. (2018). Relational mobility predicts social behaviors in 39 countries and is tied to 
historical farming and threat. Proceedings of the National Academy of Sciences, 115(29), 7521-7526. https://doi.org/10.1073/pnas.1713191115

United Nations, Department of Economic and Social Affairs, Population Division. (2018). World Population Prospects: The 2019 Revision, custom data acquired via website.

Van Bavel, J., et al. (2020). Using social and behavioural science to support COVID-19 pandemic response. Nature Human Behaviour.

Yuki, M., \& Schug, J. (2020). Psychological consequences of relational mobility. Current Opinion in Psychology, 32, 129-132. https://doi.org/10.1016/j.copsyc.2019.07.029 
Table 1.

Regression coefficients predicting the number of confirmed cases $(A)$ and deaths $(B)$ over the first 30 days of country-wise outbreaks. Models vary in the covariates included. Model 1 includes only total population (called Population). Model 2 includes all demographic covariates. Model 3 includes only those that prove at least marginally significant in Model 2 . The results are based on all 39 countries, except in Model 2, which is based on 38 countries because of a missing value for Tourism in one of the countries (Taiwan).

\begin{tabular}{|c|c|c|c|c|c|c|c|c|c|}
\hline \multirow[b]{2}{*}{ A. Predictor } & \multicolumn{3}{|c|}{ Model 1} & \multicolumn{3}{|c|}{ Model 2} & \multicolumn{3}{|c|}{ Model 3} \\
\hline & b & $\mathrm{t}$ & $\mathrm{p}$ & b & $\mathrm{t}$ & $\mathrm{p}$ & b & $\mathrm{t}$ & $\mathrm{p}$ \\
\hline Intercept & 7.023 & 20.394 & $<.001$ & 6.924 & 45.445 & 0.002 & 6.916 & 38.220 & $<.001$ \\
\hline Day & 0.131 & 6.109 & 0.005 & 0.120 & 17.055 & 0.011 & 0.121 & 12.830 & 0.006 \\
\hline $\begin{array}{l}\text { Relational } \\
\text { Mobility }\end{array}$ & 2.312 & 3.268 & 0.002 & 1.446 & 2.095 & 0.055 & 1.798 & 2.791 & 0.010 \\
\hline Population & 0.647 & 4.777 & $<.001$ & 0.351 & 2.493 & 0.019 & 0.356 & 2.744 & 0.011 \\
\hline Migration & & & & 0.263 & 2.090 & 0.046 & 0.248 & 2.171 & 0.038 \\
\hline GDP & & & & 0.103 & 0.433 & 0.668 & & & \\
\hline $\begin{array}{l}\text { Population } \\
\text { Density }\end{array}$ & & & & -0.360 & -2.161 & 0.039 & -0.251 & -2.139 & 0.040 \\
\hline Tourism & & & & 0.322 & 2.218 & 0.035 & 0.339 & 2.521 & 0.017 \\
\hline $\begin{array}{l}\text { Percent } \\
\text { Urban }\end{array}$ & & & & 0.033 & 0.231 & 0.819 & & & \\
\hline Median Age & & & & 0.115 & 0.658 & 0.518 & & & \\
\hline $\begin{array}{l}\text { Day x } \\
\text { Relational } \\
\text { Mobility }\end{array}$ & 0.131 & 3.610 & 0.001 & 0.082 & 2.514 & 0.026 & 0.112 & 3.513 & 0.002 \\
\hline $\begin{array}{l}\text { Day } \mathrm{x} \\
\text { Population }\end{array}$ & 0.039 & 5.411 & $<.001$ & 0.022 & 3.233 & 0.003 & 0.021 & 3.333 & 0.003 \\
\hline $\begin{array}{l}\text { Day } \mathrm{x} \\
\text { Migration }\end{array}$ & & & & 0.013 & 2.197 & 0.037 & 0.014 & 2.532 & 0.017 \\
\hline Day x GDP & & & & 0.016 & 1.398 & 0.173 & & & \\
\hline $\begin{array}{l}\text { Day x } \\
\text { Population } \\
\text { Density }\end{array}$ & & & & -0.014 & -1.732 & 0.094 & -0.004 & -0.726 & 0.473 \\
\hline $\begin{array}{l}\text { Day } \mathrm{x} \\
\text { Tourism }\end{array}$ & & & & 0.022 & 3.197 & 0.003 & 0.024 & 3.586 & 0.001 \\
\hline $\begin{array}{l}\text { Day x Percent } \\
\text { Urban }\end{array}$ & & & & -0.003 & -0.375 & 0.710 & & & \\
\hline $\begin{array}{l}\text { Day } \times \text { Median } \\
\text { Age }\end{array}$ & & & & 0.001 & 0.157 & 0.877 & & & \\
\hline $\begin{array}{l}R^{2} \text { fixed } \\
\text { effects }\end{array}$ & & 0.615 & & & 0.767 & & & 0.751 & \\
\hline $\begin{array}{l}R^{2} \text { fixed and } \\
\text { random } \\
\text { effects }\end{array}$ & & 0.978 & & & 0.973 & & & 0.972 & \\
\hline
\end{tabular}




\begin{tabular}{|c|c|c|c|c|c|c|c|c|c|}
\hline \multirow[b]{2}{*}{ B. Predictor } & \multicolumn{3}{|c|}{ Model 1} & \multicolumn{3}{|c|}{ Model 2} & \multicolumn{3}{|c|}{ Model 3} \\
\hline & b & $\mathrm{t}$ & $\mathrm{p}$ & b & $\mathrm{t}$ & $\mathrm{p}$ & b & $\mathrm{t}$ & $\mathrm{p}$ \\
\hline Intercept & 2.618 & 7.230 & 0.002 & 2.665 & 8.513 & 0.018 & 2.618 & 7.230 & 0.002 \\
\hline Day & 0.149 & 6.743 & 0.002 & 0.149 & 8.592 & 0.002 & 0.149 & 6.743 & 0.002 \\
\hline $\begin{array}{l}\text { Relational } \\
\text { Mobility }\end{array}$ & 2.073 & 1.860 & 0.073 & 1.689 & 1.392 & 0.189 & 2.073 & 1.860 & 0.073 \\
\hline Population & 0.472 & 2.320 & 0.027 & 0.361 & 1.455 & 0.158 & 0.472 & 2.320 & 0.027 \\
\hline Migration & & & & 0.225 & 1.037 & 0.309 & & & \\
\hline GDP & & & & 0.234 & 0.569 & 0.574 & & & \\
\hline $\begin{array}{l}\text { Population } \\
\text { Density }\end{array}$ & & & & -0.361 & -1.254 & 0.220 & & & \\
\hline Tourism & & & & 0.119 & 0.475 & 0.638 & & & \\
\hline Percent Urban & & & & 0.112 & 0.459 & 0.650 & & & \\
\hline Median Age & & & & 0.220 & 0.721 & 0.481 & & & \\
\hline $\begin{array}{l}\text { Day x } \\
\text { Relational } \\
\text { Mobility }\end{array}$ & 0.144 & 2.727 & 0.010 & 0.103 & 1.889 & 0.070 & 0.144 & 2.727 & 0.010 \\
\hline $\begin{array}{l}\text { Day } \mathrm{x} \\
\text { Population }\end{array}$ & 0.031 & 3.172 & 0.004 & 0.024 & 2.223 & 0.036 & 0.031 & 3.172 & 0.004 \\
\hline $\begin{array}{l}\text { Day } \mathrm{x} \\
\text { Migration }\end{array}$ & & & & 0.013 & 1.411 & 0.170 & & & \\
\hline Day x GDP & & & & 0.016 & 0.933 & 0.358 & & & \\
\hline $\begin{array}{l}\text { Day x } \\
\text { Population } \\
\text { Density }\end{array}$ & & & & -0.020 & -1.638 & 0.113 & & & \\
\hline Day x Tourism & & & & 0.011 & 1.026 & 0.314 & & & \\
\hline $\begin{array}{l}\text { Day x Percent } \\
\text { Urban }\end{array}$ & & & & 0.001 & 0.142 & 0.888 & & & \\
\hline $\begin{array}{l}\text { Day x Median } \\
\text { Age }\end{array}$ & & & & 0.018 & 1.335 & 0.192 & & & \\
\hline$R^{2}$ fixed effects & & 0.499 & & & 0.577 & & & 0.499 & \\
\hline $\begin{array}{l}\mathrm{R}^{2} \text { fixed and } \\
\text { random effects }\end{array}$ & & 0.969 & & & 0.970 & & & 0.969 & \\
\hline
\end{tabular}


Figure 1.

The rate of growth of confirmed cases and deaths of COVID-19 during the first 30 days of country-wise outbreaks. A. Growth curves on a log scale for confirmed cases. The solid red (or blue) line designates the growth estimated for the country one SD above (or below) the grand mean of RM. Blurred red (or blue) lines show individual countries that are higher (or lower) than the RM grand mean. B. Country-wise growth rates of confirmed cases as a function of RM. C. Growth curves on a log scale for deaths. D. Country-wise growth rates of deaths as a function of RM.
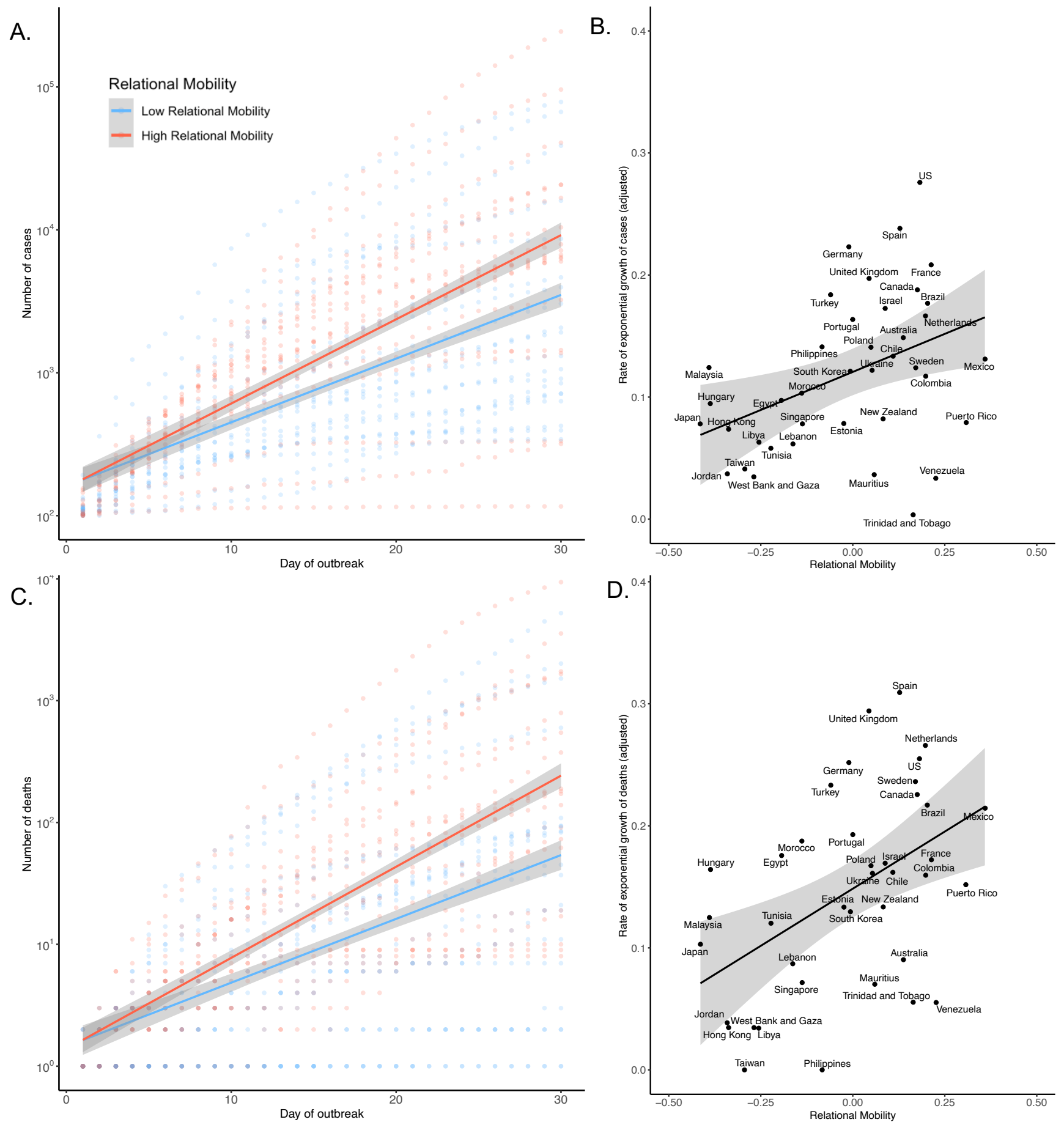
Table S1.

\section{Supplementary Tables}

Countries included in the analyses of confirmed cases and deaths. For each country, the date of the first 100 confirmed cases, the date of the first death, and the date of lockdown are noted. NI stands for not implemented.

\begin{tabular}{|c|c|c|c|}
\hline Country & $\begin{array}{c}\text { Date of first } 100 \\
\text { cases }\end{array}$ & Date of first death & Date of lockdown \\
\hline Australia & 3.10 .20 & 3.1 .20 & 3.23 .20 \\
\hline Brazil & 3.13 .20 & 3.17 .20 & 3.24 .20 \\
\hline Canada & 3.11 .20 & 3.9 .20 & $\mathrm{NI}$ \\
\hline Chile & 3.16 .20 & 3.22 .20 & 3.19 .20 \\
\hline Colombia & 3.18 .20 & 3.22 .20 & 3.25 .20 \\
\hline Egypt & 3.14 .20 & 3.8 .20 & 3.19 .20 \\
\hline Estonia & 3.14 .20 & 3.25 .20 & 3.27 .20 \\
\hline France & 2.29 .20 & 2.15 .20 & 3.17 .20 \\
\hline Germany & 3.1 .20 & 3.9 .20 & 3.23 .20 \\
\hline Hong Kong & 3.2 .20 & 2.4 .20 & $\mathrm{NI}$ \\
\hline Hungary & 3.21 .20 & 3.15 .20 & 3.28 .20 \\
\hline Israel & 3.12 .20 & 3.21 .20 & 4.02 .20 \\
\hline Japan & 2.21 .20 & 2.13 .20 & 4.07 .20 \\
\hline Jordan & 3.22 .20 & 3.27 .20 & 3.18 .20 \\
\hline Lebanon & 3.15 .20 & 3.10 .20 & 3.15 .20 \\
\hline Libya & 5.28 .20 & 4.2 .20 & 3.22 .20 \\
\hline Malaysia & 3.9 .20 & 3.17 .20 & 3.18 .20 \\
\hline Mauritius & 3.28 .20 & 3.21 .20 & 3.24 .20 \\
\hline Mexico & 3.18 .20 & 3.19 .20 & 3.26 .20 \\
\hline Morocco & 3.22 .20 & 3.10 .20 & 3.19 .20 \\
\hline
\end{tabular}




\begin{tabular}{|c|c|c|c|}
\hline Netherlands & 3.6 .20 & 3.6 .20 & 3.16 .20 \\
\hline New Zealand & 3.22 .20 & 3.29 .20 & 3.26 .20 \\
\hline Philippines & 3.14 .20 & 2.2 .20 & 3.16 .20 \\
\hline Poland & 3.14 .20 & 3.12 .20 & 3.13 .20 \\
\hline Portugal & 3.13 .20 & 3.17 .20 & 3.19 .20 \\
\hline Puerto Rico & 3.28 .20 & 3.21 .20 & 3.15 .20 \\
\hline Singapore & 2.29 .20 & 3.21 .20 & 4.07.20 \\
\hline South Korea & 2.20 .20 & 2.20 .20 & $\mathrm{NI}$ \\
\hline Spain & 3.2 .20 & 3.3 .20 & 3.14 .20 \\
\hline Sweden & 3.6 .20 & 3.11 .20 & $\mathrm{NI}$ \\
\hline Taiwan & 3.18 .20 & 2.16 .20 & $\mathrm{NI}$ \\
\hline $\begin{array}{c}\text { Trinidad and } \\
\text { Tobago }\end{array}$ & 4.4 .20 & 3.25 .20 & 3.17 .20 \\
\hline Tunisia & 3.24 .20 & 3.19 .20 & 3.22 .20 \\
\hline Turkey & 3.19 .20 & 3.17 .20 & 4.11 .20 \\
\hline Ukraine & 3.25 .20 & 3.13 .20 & $\mathrm{NI}$ \\
\hline United Kingdom & 3.2 .20 & 3.5 .20 & 3.23 .20 \\
\hline United States & 3.4 .20 & 2.29 .20 & 3.19 .20 (CA) \\
\hline Venezuela & 3.26 .20 & 3.27 .20 & 3.17 .20 \\
\hline $\begin{array}{c}\text { West Bank and } \\
\text { Gaza }\end{array}$ & 3.29 .20 & 3.26 .20 & 3.22 .20 \\
\hline
\end{tabular}


Table S2.

Regression coefficients predicting the number of confirmed cases $(A)$ and deaths $(B)$ over the first 30 days of country-wise outbreaks. Individualism is added as a covariate. Model 1 includes total population (called Population) and Individualism. Model 2 includes all demographic covariates and Individualism. Model 3 includes only those demographic variables that prove at least marginally significant in Model 2 and Individualism. The results are based on 35 of the 39 countries, except in Model 2, which is based on 34 countries because of a missing value for Tourism in Taiwan.

\begin{tabular}{|c|c|c|c|c|c|c|c|c|c|}
\hline \multirow[b]{2}{*}{ A. Predictor } & \multicolumn{3}{|c|}{ Model 1} & \multicolumn{3}{|c|}{ Model 2} & \multicolumn{3}{|c|}{ Model 3} \\
\hline & $b$ & $\mathrm{t}$ & $p$ & $b$ & $\mathrm{t}$ & $\mathrm{p}$ & $\mathrm{b}$ & $\mathrm{t}$ & $p$ \\
\hline Intercept & 6.778 & 24.778 & $<.001$ & 6.712 & 23.311 & $<.001$ & 6.731 & 27.782 & $<.001$ \\
\hline Day & 0.116 & 9.698 & 0.005 & 0.114 & 11.987 & 0.003 & 0.115 & 14.026 & 0.002 \\
\hline $\begin{array}{l}\text { Relational } \\
\text { Mobility }\end{array}$ & 1.815 & 2.386 & 0.024 & 1.697 & 2.443 & 0.022 & 1.881 & 2.751 & 0.011 \\
\hline Individualism & 0.146 & 0.973 & 0.339 & -0.272 & -1.608 & 0.122 & -0.005 & -0.036 & 0.972 \\
\hline Population & 0.891 & 4.744 & $<.001$ & 0.734 & 3.554 & 0.002 & 0.817 & 4.155 & $<.001$ \\
\hline Migration & & & & 0.542 & 3.338 & 0.003 & 0.498 & 2.982 & 0.006 \\
\hline GDP & & & & 0.180 & 0.715 & 0.482 & & & \\
\hline $\begin{array}{l}\text { Population } \\
\text { Density }\end{array}$ & & & & -0.420 & -2.299 & 0.032 & & & \\
\hline Tourism & & & & 0.152 & 1.078 & 0.293 & 0.140 & 1.007 & 0.323 \\
\hline Percent Urban & & & & 0.184 & 1.419 & 0.170 & & & \\
\hline Median Age & & & & -0.054 & -0.304 & 0.764 & & & \\
\hline $\begin{array}{l}\text { Day x Relational } \\
\text { Mobility }\end{array}$ & 0.088 & 2.372 & 0.026 & 0.077 & 2.280 & 0.034 & 0.084 & 2.869 & 0.010 \\
\hline $\begin{array}{l}\text { Day x } \\
\text { Individualism }\end{array}$ & 0.018 & 2.405 & 0.023 & 0.005 & 0.547 & 0.589 & 0.010 & 1.590 & 0.124 \\
\hline Day x Population & 0.049 & 5.271 & $<.001$ & 0.040 & 3.748 & 0.001 & 0.038 & 4.186 & $<.001$ \\
\hline Day $x$ Migration & & & & 0.022 & 2.553 & 0.019 & 0.024 & 3.053 & 0.005 \\
\hline Day x GDP & & & & 0.010 & 0.763 & 0.453 & & & \\
\hline $\begin{array}{l}\text { Day x Population } \\
\text { Density }\end{array}$ & & & & -0.007 & -0.729 & 0.474 & & & \\
\hline Day x Tourism & & & & 0.015 & 2.076 & 0.049 & 0.017 & 2.757 & 0.010 \\
\hline $\begin{array}{l}\text { Day x Percent } \\
\text { Urban }\end{array}$ & & & & 0.001 & 0.138 & 0.892 & & & \\
\hline $\begin{array}{l}\text { Day x Median } \\
\text { Age }\end{array}$ & & & & -0.001 & -0.097 & 0.924 & & & \\
\hline$R^{2}$ fixed effects & & 0.671 & & & 0.733 & & & 0.747 & \\
\hline $\mathrm{R}^{2}$ fixed and & & 0.973 & & & 0.974 & & & 0.972 & \\
\hline
\end{tabular}




\begin{tabular}{|c|c|c|c|c|c|c|c|c|c|}
\hline \multirow[b]{2}{*}{ B. Predictor } & \multicolumn{3}{|c|}{ Model 1} & \multicolumn{3}{|c|}{ Model 2} & \multicolumn{3}{|c|}{ Model 3} \\
\hline & $\mathrm{b}$ & $\mathrm{t}$ & $p$ & $\mathrm{~b}$ & $\mathrm{t}$ & $\mathrm{p}$ & $b$ & $\mathrm{t}$ & $\mathrm{p}$ \\
\hline Intercept & 2.519 & 6.478 & 0.003 & 2.399 & 5.354 & 0.007 & 2.448 & 6.049 & 0.003 \\
\hline Day & 0.140 & 7.202 & 0.001 & 0.137 & 6.480 & 0.003 & 0.136 & 6.728 & 0.002 \\
\hline $\begin{array}{l}\text { Relational } \\
\text { Mobility }\end{array}$ & 1.725 & 1.378 & 0.180 & 0.578 & 0.445 & 0.660 & 1.831 & 1.473 & 0.152 \\
\hline Individualism & 0.105 & 0.424 & 0.675 & -0.579 & -1.804 & 0.084 & -0.014 & -0.054 & 0.957 \\
\hline Population & 0.686 & 2.137 & 0.041 & 0.810 & 2.048 & 0.052 & 0.710 & 2.265 & 0.031 \\
\hline Migration & & & & 0.381 & 1.215 & 0.238 & 0.465 & 1.490 & 0.147 \\
\hline GDP & & & & 0.874 & 1.813 & 0.083 & & & \\
\hline $\begin{array}{l}\text { Population } \\
\text { Density }\end{array}$ & & & & -0.781 & -2.217 & 0.038 & & & \\
\hline Tourism & & & & -0.048 & -0.177 & 0.861 & & & \\
\hline Percent Urban & & & & 0.295 & 1.194 & 0.245 & & & \\
\hline Median Age & & & & -0.071 & -0.212 & 0.834 & & & \\
\hline $\begin{array}{l}\text { Day x } \\
\text { Relational } \\
\text { Mobility }\end{array}$ & 0.094 & 1.708 & 0.098 & 0.075 & 1.312 & 0.202 & 0.101 & 1.930 & 0.063 \\
\hline $\begin{array}{l}\text { Day x } \\
\text { Individualism }\end{array}$ & 0.024 & 2.175 & 0.038 & 0.002 & 0.114 & 0.910 & 0.016 & 1.533 & 0.136 \\
\hline $\begin{array}{l}\text { Day } \mathrm{x} \\
\text { Population }\end{array}$ & 0.043 & 3.115 & 0.004 & 0.045 & 2.636 & 0.015 & 0.044 & 3.457 & 0.002 \\
\hline Day x Migration & & & & 0.024 & 1.783 & 0.089 & 0.028 & 2.210 & 0.036 \\
\hline Day $x$ GDP & & & & 0.019 & 0.887 & 0.385 & & & \\
\hline $\begin{array}{l}\text { Day x } \\
\text { Population } \\
\text { Density }\end{array}$ & & & & -0.017 & -1.117 & 0.276 & & & \\
\hline Day x Tourism & & & & 0.004 & 0.315 & 0.755 & & & \\
\hline $\begin{array}{l}\text { Day x Percent } \\
\text { Urban }\end{array}$ & & & & 0.005 & 0.509 & 0.616 & & & \\
\hline $\begin{array}{l}\text { Day } x \text { Median } \\
\text { Age }\end{array}$ & & & & 0.011 & 0.741 & 0.466 & & & \\
\hline$R^{2}$ fixed effects & & 0.491 & & & 0.535 & & & 0.504 & \\
\hline $\begin{array}{l}R^{2} \text { fixed and } \\
\text { random effects }\end{array}$ & & 0.967 & & & 0.970 & & & 0.968 & \\
\hline
\end{tabular}


Table S3.

Regression coefficients predicting the number of confirmed cases $(A)$ and deaths $(B)$ over the first 30 days of country-wise outbreaks. Government efficiency is added as a covariate. Model 1 includes total population (called Population) and Government efficiency. Model 2 includes all demographic covariates and Government efficiency. Model 3 includes only those demographic variables that prove at least marginally significant in Model 2 and Government efficiency. The results are based on 34 of the 39 countries.

\begin{tabular}{|c|c|c|c|c|c|c|c|c|c|}
\hline \multirow[b]{2}{*}{ A. Predictor } & \multicolumn{3}{|c|}{ Model 1} & \multicolumn{3}{|c|}{ Model 2} & \multicolumn{3}{|c|}{ Model 3} \\
\hline & $b$ & $\mathrm{t}$ & $\mathrm{p}$ & $b$ & $\mathrm{t}$ & $\mathrm{p}$ & $b$ & $\mathrm{t}$ & $\mathrm{p}$ \\
\hline Intercept & 7.029 & 21.247 & $<.001$ & 6.888 & 28.652 & 0.002 & 6.886 & 28.180 & $<.001$ \\
\hline Day & 0.131 & 6.725 & 0.008 & 0.120 & 11.164 & 0.021 & 0.121 & 10.112 & 0.005 \\
\hline $\begin{array}{l}\text { Relational } \\
\text { Mobility }\end{array}$ & 2.279 & 2.916 & 0.007 & 1.490 & 1.865 & 0.075 & 1.951 & 2.791 & 0.009 \\
\hline $\begin{array}{l}\text { Government } \\
\text { Efficiency }\end{array}$ & 0.174 & 1.125 & 0.270 & -0.203 & -0.841 & 0.410 & -0.224 & -1.200 & 0.240 \\
\hline Population & 0.663 & 4.488 & $<.001$ & 0.347 & 2.177 & 0.041 & 0.444 & 3.045 & 0.006 \\
\hline Migration & & & & 0.644 & 2.780 & 0.012 & 0.655 & 2.939 & 0.007 \\
\hline GDP & & & & 0.109 & 0.380 & 0.708 & & & \\
\hline $\begin{array}{l}\text { Population } \\
\text { Density }\end{array}$ & & & & -0.313 & -1.829 & 0.082 & & & \\
\hline Tourism & & & & 0.271 & 1.830 & 0.081 & 0.247 & 1.716 & 0.097 \\
\hline Percent Urban & & & & 0.111 & 0.784 & 0.441 & & & \\
\hline Median Age & & & & -0.084 & -0.412 & 0.684 & & & \\
\hline $\begin{array}{l}\text { Day } \times \text { Relational } \\
\text { Mobility }\end{array}$ & 0.133 & 3.570 & 0.001 & 0.085 & 2.174 & 0.040 & 0.116 & 3.529 & 0.001 \\
\hline $\begin{array}{l}\text { Day } x \\
\text { Government } \\
\text { Efficiency }\end{array}$ & 0.016 & 2.131 & 0.042 & -0.008 & -0.662 & 0.515 & -0.002 & -0.267 & 0.791 \\
\hline Day x Population & 0.041 & 5.562 & $<.001$ & 0.022 & 2.844 & 0.010 & 0.026 & 3.721 & 0.001 \\
\hline Day x Migration & & & & 0.028 & 2.435 & 0.025 & 0.027 & 2.620 & 0.015 \\
\hline Day $x$ GDP & & & & 0.017 & 1.202 & 0.243 & & & \\
\hline $\begin{array}{l}\text { Day x Population } \\
\text { Density }\end{array}$ & & & & -0.012 & -1.416 & 0.172 & & & \\
\hline Day x Tourism & & & & 0.019 & 2.623 & 0.015 & 0.019 & 2.836 & 0.008 \\
\hline $\begin{array}{l}\text { Day x Percent } \\
\text { Urban }\end{array}$ & & & & 0.001 & 0.078 & 0.939 & & & \\
\hline $\begin{array}{l}\text { Day } x \text { Median } \\
\text { Age }\end{array}$ & & & & -0.006 & -0.567 & 0.576 & & & \\
\hline$R^{2}$ fixed effects & & 0.634 & & & 0.739 & & & 0.731 & \\
\hline $\begin{array}{l}\mathrm{R}^{2} \text { fixed and } \\
\text { random effects }\end{array}$ & & 0.977 & & & 0.974 & & & 0.974 & \\
\hline
\end{tabular}




\begin{tabular}{|c|c|c|c|c|c|c|c|c|c|}
\hline \multirow[b]{2}{*}{ B. Predictor } & \multicolumn{3}{|c|}{ Model 1} & \multicolumn{3}{|c|}{ Model 2} & \multicolumn{3}{|c|}{ Model 3} \\
\hline & $b$ & $\mathrm{t}$ & $\mathrm{p}$ & b & $\mathrm{t}$ & $\mathrm{p}$ & b & $\mathrm{t}$ & $\mathrm{p}$ \\
\hline Intercept & 2.752 & 7.174 & 0.002 & 2.687 & 7.577 & 0.006 & 2.752 & 7.174 & 0.002 \\
\hline Day & 0.155 & 6.542 & 0.003 & 0.151 & 7.543 & 0.003 & 0.155 & 6.542 & 0.003 \\
\hline Relational Mobility & 1.499 & 1.254 & 0.220 & 0.215 & 0.152 & 0.881 & 1.499 & 1.254 & 0.220 \\
\hline $\begin{array}{l}\text { Government } \\
\text { Efficiency }\end{array}$ & 0.224 & 0.940 & 0.355 & -0.388 & -0.886 & 0.385 & 0.224 & 0.940 & 0.355 \\
\hline Population & 0.479 & 2.231 & 0.035 & 0.170 & 0.610 & 0.548 & 0.479 & 2.231 & 0.035 \\
\hline Migration & & & & 0.543 & 1.281 & 0.214 & & & \\
\hline GDP & & & & 0.573 & 1.094 & 0.285 & & & \\
\hline Population Density & & & & -0.509 & -1.635 & 0.116 & & & \\
\hline Tourism & & & & 0.154 & 0.575 & 0.571 & & & \\
\hline Percent Urban & & & & 0.221 & 0.862 & 0.397 & & & \\
\hline Median Age & & & & -0.127 & -0.355 & 0.726 & & & \\
\hline $\begin{array}{l}\text { Day } x \text { Relational } \\
\text { Mobility }\end{array}$ & 0.115 & 2.257 & 0.032 & 0.084 & 1.402 & 0.174 & 0.115 & 2.257 & 0.032 \\
\hline $\begin{array}{l}\text { Day x Government } \\
\text { Efficiency }\end{array}$ & 0.027 & 2.672 & 0.012 & 0.013 & 0.709 & 0.486 & 0.027 & 2.672 & 0.012 \\
\hline Day x Population & 0.038 & 3.868 & 0.001 & 0.027 & 2.221 & 0.037 & 0.038 & 3.868 & 0.001 \\
\hline Day $x$ Migration & & & & 0.017 & 1.004 & 0.327 & & & \\
\hline Day x GDP & & & & 0.008 & 0.384 & 0.705 & & & \\
\hline $\begin{array}{l}\text { Day } \times \text { Population } \\
\text { Density }\end{array}$ & & & & -0.018 & -1.424 & 0.169 & & & \\
\hline Day x Tourism & & & & 0.011 & 1.010 & 0.323 & & & \\
\hline $\begin{array}{l}\text { Day x Percent } \\
\text { Urban }\end{array}$ & & & & 0.002 & 0.157 & 0.876 & & & \\
\hline Day x Median Age & & & & 0.013 & 0.858 & 0.400 & & & \\
\hline$R^{2}$ fixed effects & & 0.507 & & & 0.549 & & & 0.507 & \\
\hline $\begin{array}{l}\mathrm{R}^{2} \text { fixed and } \\
\text { random effects }\end{array}$ & & 0.967 & & & 0.968 & & & 0.967 & \\
\hline
\end{tabular}


Table S4.

Regression coefficients predicting the number of confirmed cases $(A)$ and deaths $(B)$ over the first 30 days of country-wise outbreaks. Tightness of cultural norms is added as a covariate. Model 1 includes total population (called Population) and Tightness. Model 2 includes all demographic covariates and Tightness. Model 3 includes only those demographic variables that prove at least marginally significant in Model 2 and Tightness. The results are based on 24 of the 39 countries.

\begin{tabular}{|c|c|c|c|c|c|c|c|c|c|}
\hline \multirow[b]{2}{*}{ A. Predictor } & \multicolumn{3}{|c|}{ Model 1} & \multicolumn{3}{|c|}{ Model 2} & \multicolumn{3}{|c|}{ Model 3} \\
\hline & b & $\mathrm{t}$ & $\mathrm{p}$ & b & $\mathrm{t}$ & $\mathrm{p}$ & $b$ & $\mathrm{t}$ & 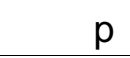 \\
\hline Intercept & 7.105 & 21.300 & $<.001$ & 6.966 & 19.562 & $<.001$ & 7.105 & 21.298 & $<.001$ \\
\hline Day & 0.132 & 6.685 & 0.003 & 0.116 & 6.664 & 0.002 & 0.132 & 6.685 & 0.003 \\
\hline $\begin{array}{l}\text { Relational } \\
\text { Mobility }\end{array}$ & 1.562 & 1.816 & 0.084 & 1.471 & 1.490 & 0.159 & 1.562 & 1.817 & 0.084 \\
\hline $\begin{array}{l}\text { Tightness- } \\
\text { Looseness }\end{array}$ & 0.089 & 0.518 & 0.610 & 0.196 & 0.921 & 0.373 & 0.089 & 0.518 & 0.610 \\
\hline Population & 0.975 & 4.632 & $<.001$ & 0.483 & 1.241 & 0.237 & 0.975 & 4.632 & $<.001$ \\
\hline Migration & & & & 0.479 & 0.936 & 0.366 & & & \\
\hline GDP & & & & -0.220 & -0.543 & 0.596 & & & \\
\hline $\begin{array}{l}\text { Population } \\
\text { Density }\end{array}$ & & & & -0.204 & -0.795 & 0.441 & & & \\
\hline Tourism & & & & 0.355 & 1.440 & 0.175 & & & \\
\hline Percent Urban & & & & 0.233 & 0.737 & 0.474 & & & \\
\hline Median Age & & & & -0.060 & -0.228 & 0.823 & & & \\
\hline $\begin{array}{l}\text { Day x } \\
\text { Relational } \\
\text { Mobility }\end{array}$ & 0.087 & 1.957 & 0.065 & 0.057 & 1.266 & 0.227 & 0.087 & 1.957 & 0.065 \\
\hline $\begin{array}{l}\text { Day x } \\
\text { Tightness- } \\
\text { Looseness }\end{array}$ & -0.001 & -0.165 & 0.870 & -0.007 & -0.780 & 0.450 & -0.001 & -0.165 & 0.870 \\
\hline $\begin{array}{l}\text { Day } \mathrm{x} \\
\text { Population }\end{array}$ & 0.054 & 5.070 & $<.001$ & 0.039 & 2.211 & 0.047 & 0.054 & 5.070 & $<.001$ \\
\hline Day $x$ Migration & & & & 0.029 & 1.247 & 0.235 & & & \\
\hline Day x GDP & & & & 0.015 & 0.838 & 0.417 & & & \\
\hline $\begin{array}{l}\text { Day x } \\
\text { Population } \\
\text { Density }\end{array}$ & & & & -0.012 & -1.009 & 0.332 & & & \\
\hline Day x Tourism & & & & 0.018 & 1.633 & 0.129 & & & \\
\hline $\begin{array}{l}\text { Day x Percent } \\
\text { Urban }\end{array}$ & & & & 0.004 & 0.312 & 0.760 & & & \\
\hline $\begin{array}{l}\text { Day } x \text { Median } \\
\text { Age }\end{array}$ & & & & -0.006 & -0.482 & 0.637 & & & \\
\hline$R^{2}$ fixed effects & & 0.679 & & & 0.701 & & & 0.679 & \\
\hline $\begin{array}{l}\mathrm{R}^{2} \text { fixed and } \\
\text { random effects }\end{array}$ & & 0.973 & & & 0.973 & & & 0.973 & \\
\hline
\end{tabular}




\begin{tabular}{|c|c|c|c|c|c|c|c|c|c|}
\hline \multirow[b]{2}{*}{ B. Predictor } & \multicolumn{3}{|c|}{ Model 1} & \multicolumn{3}{|c|}{ Model 2} & \multicolumn{3}{|c|}{ Model 3} \\
\hline & b & $t$ & $\mathrm{p}$ & b & $\mathrm{t}$ & $\mathrm{p}$ & b & $\mathrm{t}$ & $\mathrm{p}$ \\
\hline Intercept & 2.976 & 7.610 & 0.001 & 3.103 & 6.341 & 0.001 & 3.029 & 8.124 & 0.004 \\
\hline Day & 0.158 & 6.987 & 0.001 & 0.153 & 6.346 & 0.002 & 0.157 & 7.222 & 0.002 \\
\hline $\begin{array}{l}\text { Relational } \\
\text { Mobility }\end{array}$ & 1.470 & 1.203 & 0.243 & 1.179 & 0.856 & 0.407 & 1.456 & 1.215 & 0.239 \\
\hline $\begin{array}{l}\text { Tightness- } \\
\text { Looseness }\end{array}$ & 0.052 & 0.212 & 0.834 & 0.105 & 0.352 & 0.730 & 0.011 & 0.048 & 0.962 \\
\hline Population & 0.863 & 2.865 & 0.010 & 0.190 & 0.349 & 0.733 & & & \\
\hline Migration & & & & -0.274 & -0.383 & 0.708 & & & \\
\hline GDP & & & & 0.034 & 0.060 & 0.953 & & & \\
\hline $\begin{array}{l}\text { Population } \\
\text { Density }\end{array}$ & & & & -0.248 & -0.691 & 0.501 & & & \\
\hline Tourism & & & & 0.529 & 1.532 & 0.151 & 0.662 & 3.049 & 0.007 \\
\hline $\begin{array}{l}\text { Percent } \\
\text { Urban }\end{array}$ & & & & 0.407 & 0.920 & 0.374 & 0.274 & 0.943 & 0.358 \\
\hline Median Age & & & & -0.267 & -0.720 & 0.484 & & & \\
\hline $\begin{array}{l}\text { Day x } \\
\text { Relational } \\
\text { Mobility }\end{array}$ & 0.092 & 1.823 & 0.084 & 0.054 & 1.232 & 0.241 & 0.071 & 1.581 & 0.131 \\
\hline $\begin{array}{l}\text { Day x } \\
\text { Tightness- } \\
\text { Looseness }\end{array}$ & -0.000 & -0.028 & 0.978 & 0.001 & 0.067 & 0.948 & -0.002 & -0.213 & 0.834 \\
\hline $\begin{array}{l}\text { Day } \mathrm{x} \\
\text { Population }\end{array}$ & 0.051 & 4.299 & $<.001$ & 0.019 & 1.141 & 0.277 & & & \\
\hline $\begin{array}{l}\text { Day } \mathrm{x} \\
\text { Migration }\end{array}$ & & & & -0.006 & -0.260 & 0.799 & & & \\
\hline Day x GDP & & & & 0.009 & 0.485 & 0.637 & & & \\
\hline $\begin{array}{l}\text { Day x } \\
\text { Population } \\
\text { Density }\end{array}$ & & & & -0.017 & -1.566 & 0.144 & & & \\
\hline $\begin{array}{l}\text { Day } \mathrm{x} \\
\text { Tourism }\end{array}$ & & & & 0.021 & 2.040 & 0.066 & 0.033 & 4.148 & 0.001 \\
\hline $\begin{array}{l}\text { Day } x \\
\text { Percent } \\
\text { Urban }\end{array}$ & & & & 0.036 & 2.539 & 0.025 & 0.034 & 3.197 & 0.005 \\
\hline $\begin{array}{l}\text { Day } x \\
\text { Median Age }\end{array}$ & & & & -0.005 & -0.456 & 0.656 & & & \\
\hline $\begin{array}{l}R^{2} \text { fixed } \\
\text { effects }\end{array}$ & & 0.619 & & & 0.609 & & & 0.637 & \\
\hline $\begin{array}{l}\mathrm{R}^{2} \text { fixed and } \\
\text { random } \\
\text { effects }\end{array}$ & & 0.963 & & & 0.964 & & & 0.962 & \\
\hline
\end{tabular}


Table S5.

Regression coefficients predicting the number of confirmed cases $(A)$ and deaths $(B)$ over the first 30 days of country-wise outbreaks. BCG policy status is added as a covariate. Model 1 includes total population (called Population) and BCG. Model 2 includes all demographic covariates and BCG. Model 3 includes only those demographic variables that prove at least marginally significant in Model 2 and BCG. The results are based on 37 of the 39 countries, with the exception of Model 2, which is based on 36 countries because of a missing value for Tourism in one of the countries (Taiwan).

\begin{tabular}{|c|c|c|c|c|c|c|c|c|c|}
\hline \multirow[b]{2}{*}{ A. Predictor } & \multicolumn{3}{|c|}{ Model 1} & \multicolumn{3}{|c|}{ Model 2} & \multicolumn{3}{|c|}{ Model 3} \\
\hline & b & $t$ & $\mathrm{p}$ & $b$ & $\mathrm{t}$ & $\mathrm{p}$ & b & $t$ & $\mathrm{p}$ \\
\hline Intercept & 7.217 & 19.589 & $<.001$ & 7.019 & 28.515 & $<.001$ & 7.150 & 28.089 & $<.001$ \\
\hline Day & 0.149 & 7.045 & 0.001 & 0.137 & 12.429 & $<.001$ & 0.142 & 14.268 & $<.001$ \\
\hline $\begin{array}{l}\text { Relational } \\
\text { Mobility }\end{array}$ & 1.877 & 2.254 & 0.032 & 1.462 & 2.602 & 0.015 & 1.721 & 2.705 & 0.016 \\
\hline $\begin{array}{l}\text { BCG Current } \\
\text { vs. Not Current }\end{array}$ & -0.297 & -0.933 & 0.358 & -0.105 & -0.343 & 0.734 & -0.338 & -1.194 & 0.243 \\
\hline Population & 0.558 & 3.996 & 0.001 & 0.327 & 2.705 & 0.012 & 0.405 & 3.406 & 0.002 \\
\hline Migration & & & & 0.493 & 2.902 & 0.007 & 0.484 & 3.069 & 0.005 \\
\hline GDP & & & & 0.082 & 0.346 & 0.732 & & & \\
\hline $\begin{array}{l}\text { Population } \\
\text { Density }\end{array}$ & & & & -0.330 & -1.945 & 0.063 & & & \\
\hline Tourism & & & & 0.331 & 2.603 & 0.015 & 0.304 & 2.498 & 0.019 \\
\hline Percent Urban & & & & -0.126 & -0.942 & 0.355 & -0.214 & -1.819 & 0.079 \\
\hline Median Age & & & & 0.117 & 0.800 & 0.431 & & & \\
\hline $\begin{array}{l}\text { Day } x \text { Relational } \\
\text { Mobility }\end{array}$ & 0.091 & 2.124 & 0.041 & 0.081 & 3.213 & 0.003 & 0.088 & 3.514 & 0.004 \\
\hline $\begin{array}{l}\text { Day x BCG } \\
\text { Current vs. Not } \\
\text { Current }\end{array}$ & -0.028 & -1.727 & 0.094 & -0.022 & -1.624 & 0.116 & -0.030 & -2.589 & 0.016 \\
\hline Day x Population & 0.035 & 4.678 & $<.001$ & 0.021 & 3.904 & 0.001 & 0.021 & 4.197 & $<.001$ \\
\hline Day x Migration & & & & 0.021 & 2.734 & 0.011 & 0.025 & 3.641 & 0.001 \\
\hline Day x GDP & & & & 0.010 & 0.948 & 0.352 & & & \\
\hline $\begin{array}{l}\text { Day x Population } \\
\text { Density }\end{array}$ & & & & -0.008 & -1.045 & 0.305 & & & \\
\hline Day $x$ Tourism & & & & 0.022 & 3.922 & 0.001 & 0.025 & 4.926 & $<.001$ \\
\hline $\begin{array}{l}\text { Day x Percent } \\
\text { Urban }\end{array}$ & & & & -0.012 & -2.038 & 0.052 & -0.011 & -2.230 & 0.034 \\
\hline $\begin{array}{l}\text { Day } x \text { Median } \\
\text { Age }\end{array}$ & & & & 0.002 & 0.333 & 0.742 & & & \\
\hline$R^{2}$ fixed effects & & 0.649 & & & 0.830 & & & 0.802 & \\
\hline $\begin{array}{l}\mathrm{R}^{2} \text { fixed and } \\
\text { random effects }\end{array}$ & & 0.975 & & & 0.972 & & & 0.971 & \\
\hline
\end{tabular}




\begin{tabular}{|c|c|c|c|c|c|c|c|c|c|}
\hline \multirow[b]{2}{*}{ B. Predictor } & \multicolumn{2}{|c|}{ Model 1} & \multicolumn{4}{|c|}{ Model 2} & \multicolumn{3}{|c|}{ Model 3} \\
\hline & b & $t$ & $p$ & b & $\mathrm{t}$ & $\mathrm{p}$ & b & $\mathrm{t}$ & $\mathrm{p}$ \\
\hline Intercept & 3.224 & 5.712 & $<.001$ & 2.841 & 4.465 & 0.002 & 3.087 & 5.339 & 0.001 \\
\hline Day & 0.185 & 6.546 & $<.001$ & 0.169 & 6.201 & $<.001$ & 0.175 & 6.306 & $<.001$ \\
\hline $\begin{array}{l}\text { Relational } \\
\text { Mobility }\end{array}$ & 0.974 & 0.727 & 0.473 & 0.674 & 0.465 & 0.647 & 1.072 & 0.803 & 0.429 \\
\hline $\begin{array}{l}\text { BCG Current } \\
\text { vs. Not Current }\end{array}$ & -0.764 & -1.476 & 0.150 & -0.244 & -0.366 & 0.717 & -0.635 & -1.216 & 0.233 \\
\hline Population & 0.443 & 1.993 & 0.058 & 0.336 & 1.238 & 0.231 & 0.445 & 2.013 & 0.057 \\
\hline Migration & & & & 0.290 & 0.868 & 0.395 & 0.402 & 1.367 & 0.182 \\
\hline GDP & & & & 0.363 & 0.743 & 0.464 & & & \\
\hline $\begin{array}{l}\text { Population } \\
\text { Density }\end{array}$ & & & & -0.375 & -1.104 & 0.281 & & & \\
\hline Tourism & & & & 0.127 & 0.484 & 0.633 & & & \\
\hline Percent Urban & & & & -0.010 & -0.035 & 0.973 & & & \\
\hline Median Age & & & & 0.097 & 0.288 & 0.775 & & & \\
\hline $\begin{array}{l}\text { Day x } \\
\text { Relational } \\
\text { Mobility }\end{array}$ & 0.075 & 1.224 & 0.230 & 0.055 & 0.944 & 0.354 & 0.083 & 1.440 & 0.160 \\
\hline $\begin{array}{l}\text { Day x BCG } \\
\text { Current vs. Not } \\
\text { Current }\end{array}$ & -0.047 & -2.018 & 0.052 & -0.028 & -1.077 & 0.291 & -0.037 & -1.707 & 0.098 \\
\hline $\begin{array}{l}\text { Day } \mathrm{x} \\
\text { Population }\end{array}$ & 0.030 & 2.914 & 0.008 & 0.024 & 2.193 & 0.041 & 0.031 & 3.138 & 0.005 \\
\hline $\begin{array}{l}\text { Day } \mathrm{x} \\
\text { Migration }\end{array}$ & & & & 0.023 & 1.813 & 0.083 & 0.030 & 2.464 & 0.020 \\
\hline Day $x$ GDP & & & & 0.017 & 0.899 & 0.377 & & & \\
\hline $\begin{array}{l}\text { Day x } \\
\text { Population } \\
\text { Density }\end{array}$ & & & & -0.015 & -1.122 & 0.273 & & & \\
\hline Day x Tourism & & & & 0.011 & 1.117 & 0.275 & & & \\
\hline $\begin{array}{l}\text { Day x Percent } \\
\text { Urban }\end{array}$ & & & & -0.010 & -0.886 & 0.384 & & & \\
\hline $\begin{array}{l}\text { Day x Median } \\
\text { Age }\end{array}$ & & & & 0.014 & 1.093 & 0.285 & & & \\
\hline$R^{2}$ fixed effects & & 0.483 & & & 0.528 & & & 0.500 & \\
\hline $\begin{array}{l}R^{2} \text { fixed and } \\
\text { random effects }\end{array}$ & & 0.970 & & & 0.971 & & & 0.971 & \\
\hline
\end{tabular}


Table S6-A.

Regression coefficients predicting the number of confirmed cases over the first 30 days of country-wise outbreaks, with the Russell et al. (2020) underreporting index used as a weighting factor. Model 1 includes total population (called Population). Model 2 includes all demographic covariates. Model 3 includes only those demographic variables that prove at least marginally significant in Model 2. The results are based on 29 of the 39 countries.

\begin{tabular}{|c|c|c|c|c|c|c|c|c|c|}
\hline \multirow[b]{2}{*}{ Predictor } & \multicolumn{3}{|c|}{ Model1 } & \multicolumn{3}{|c|}{ Model 2} & \multicolumn{3}{|c|}{ Model 3} \\
\hline & b & $\mathrm{t}$ & $\mathrm{p}$ & $b$ & $\mathrm{t}$ & $\mathrm{p}$ & b & $\mathrm{t}$ & $\mathrm{p}$ \\
\hline Intercept & 7.026 & 28.270 & $<.001$ & 7.342 & 20.418 & $<.001$ & 7.238 & 60.459 & $<.001$ \\
\hline Day & 0.124 & 8.003 & 0.001 & 0.138 & 8.626 & $<.001$ & 0.134 & 23.453 & $<.001$ \\
\hline $\begin{array}{l}\text { Relational } \\
\text { Mobility }\end{array}$ & 1.880 & 2.647 & 0.014 & 1.690 & 2.309 & 0.032 & 1.550 & 2.503 & 0.019 \\
\hline Population & 0.668 & 3.329 & 0.003 & 0.259 & 0.972 & 0.342 & & & \\
\hline Migration & & & & 0.239 & 1.556 & 0.135 & 0.255 & 2.034 & 0.053 \\
\hline GDP & & & & 0.192 & 0.756 & 0.458 & 0.149 & 0.900 & 0.377 \\
\hline $\begin{array}{l}\text { Population } \\
\text { Density }\end{array}$ & & & & 0.723 & 0.526 & 0.605 & & & \\
\hline Tourism & & & & 0.314 & 1.945 & 0.066 & 0.404 & 3.275 & 0.003 \\
\hline Percent Urban & & & & -0.091 & -0.493 & 0.627 & & & \\
\hline Median Age & & & & 0.069 & 0.378 & 0.710 & & & \\
\hline $\begin{array}{l}\text { Day x } \\
\text { Relational } \\
\text { Mobility }\end{array}$ & 0.121 & 3.130 & 0.004 & 0.099 & 3.053 & 0.006 & 0.095 & 3.199 & 0.004 \\
\hline $\begin{array}{l}\text { Day } \mathrm{x} \\
\text { Population }\end{array}$ & 0.044 & 4.078 & $<.001$ & 0.017 & 1.401 & 0.177 & & & \\
\hline $\begin{array}{l}\text { Day } x \\
\text { Migration }\end{array}$ & & & & 0.012 & 1.778 & 0.090 & 0.016 & 2.662 & 0.013 \\
\hline Day x GDP & & & & 0.025 & 2.208 & 0.039 & 0.013 & 1.574 & 0.128 \\
\hline $\begin{array}{l}\text { Day x } \\
\text { Population } \\
\text { Density }\end{array}$ & & & & 0.030 & 0.487 & 0.632 & & & \\
\hline Day x Tourism & & & & 0.020 & 2.705 & 0.013 & 0.026 & 4.405 & $<.001$ \\
\hline $\begin{array}{l}\text { Day x Percent } \\
\text { Urban }\end{array}$ & & & & -0.009 & -1.068 & 0.298 & & & \\
\hline $\begin{array}{l}\text { Day x Median } \\
\text { Age }\end{array}$ & & & & -0.005 & -0.645 & 0.527 & & & \\
\hline$R^{2}$ fixed effects & & 0.721 & & & 0.812 & & & 0.823 & \\
\hline $\begin{array}{l}\mathrm{R}^{2} \text { fixed and } \\
\text { random effects }\end{array}$ & & 0.991 & & & 0.991 & & & 0.991 & \\
\hline
\end{tabular}


Table S6-B.

Regression coefficients predicting the number of confirmed cases over the first 30 days of country-wise outbreaks, with the Russell et al. (2020) underreporting index used as a covariate. Model 1 includes total population (called Population) and Underreporting. Model 2 includes all demographic covariates and Underreporting. Model 3 includes only those demographic variables that prove at least marginally significant in Model 2 and Underreporting. The results are based on 29 of the 39 countries.

\begin{tabular}{|c|c|c|c|c|c|c|c|c|c|}
\hline \multirow[b]{2}{*}{ Predictor } & \multicolumn{3}{|c|}{ Model 1} & \multicolumn{3}{|c|}{ Model 2} & \multicolumn{3}{|c|}{ Model 3} \\
\hline & $\mathrm{b}$ & $\mathrm{t}$ & $\mathrm{p}$ & b & $\mathrm{t}$ & $\mathrm{p}$ & $b$ & $\mathrm{t}$ & $\mathrm{p}$ \\
\hline Intercept & 6.938 & 23.570 & $<.001$ & 7.317 & 20.067 & $<.001$ & 7.240 & 56.800 & $<.001$ \\
\hline Day & 0.120 & 6.645 & 0.003 & 0.138 & 8.319 & $<.001$ & 0.135 & 20.652 & $<.001$ \\
\hline $\begin{array}{l}\text { Relational } \\
\text { Mobility }\end{array}$ & 1.633 & 2.457 & 0.021 & 1.787 & 2.360 & 0.029 & 1.418 & 2.162 & 0.041 \\
\hline Reporting Index & 0.329 & 2.480 & 0.020 & 0.099 & 0.627 & 0.538 & 0.126 & 0.895 & 0.379 \\
\hline Population & 0.852 & 4.382 & $<.001$ & 0.274 & 1.014 & 0.323 & & & \\
\hline Migration & & & & 0.211 & 1.310 & 0.206 & & & \\
\hline GDP & & & & 0.146 & 0.544 & 0.593 & 0.122 & 0.637 & 0.530 \\
\hline $\begin{array}{l}\text { Population } \\
\text { Density }\end{array}$ & & & & 0.692 & 0.498 & 0.624 & & & \\
\hline Tourism & & & & 0.346 & 2.021 & 0.058 & 0.488 & 3.462 & 0.002 \\
\hline Percent Urban & & & & -0.122 & -0.630 & 0.536 & & & \\
\hline Median Age & & & & 0.094 & 0.494 & 0.627 & & & \\
\hline $\begin{array}{l}\text { Day x } \\
\text { Relational } \\
\text { Mobility }\end{array}$ & 0.107 & 2.937 & 0.007 & 0.098 & 2.849 & 0.010 & 0.080 & 2.389 & 0.025 \\
\hline $\begin{array}{l}\text { Day } x \text { Reporting } \\
\text { Index }\end{array}$ & 0.016 & 2.207 & 0.037 & 0.001 & 0.116 & 0.909 & 0.004 & 0.539 & 0.595 \\
\hline $\begin{array}{l}\text { Day } \mathrm{x} \\
\text { Population }\end{array}$ & 0.053 & 5.009 & $<.001$ & 0.018 & 1.455 & 0.162 & & & \\
\hline Day x Migration & & & & 0.012 & 1.661 & 0.113 & & & \\
\hline Day $x$ GDP & & & & 0.024 & 1.954 & 0.066 & 0.013 & 1.284 & 0.211 \\
\hline $\begin{array}{l}\text { Day x } \\
\text { Population } \\
\text { Density }\end{array}$ & & & & 0.032 & 0.502 & 0.621 & & & \\
\hline Day $x$ Tourism & & & & 0.020 & 2.588 & 0.018 & 0.030 & 4.182 & $<.001$ \\
\hline $\begin{array}{l}\text { Day } \mathrm{x} \text { Percent } \\
\text { Urban }\end{array}$ & & & & -0.009 & -1.033 & 0.315 & & & \\
\hline $\begin{array}{l}\text { Day x Median } \\
\text { Age }\end{array}$ & & & & -0.004 & -0.422 & 0.678 & & & \\
\hline$R^{2}$ fixed effects & & 0.690 & & & 0.788 & & & 0.777 & \\
\hline $\begin{array}{l}\mathrm{R}^{2} \text { fixed and } \\
\text { random effects }\end{array}$ & & 0.968 & & & 0.968 & & & 0.967 & \\
\hline
\end{tabular}


Table S7-A.

Regression coefficients predicting the number of confirmed cases over the first 30 days of country-wise outbreaks, with testing availability used as a weighting factor. Model 1 includes total population (called Population). Model 2 includes all demographic covariates. Model 3 includes only those demographic variables that prove at least marginally significant in Model 2. The results are based on 29 of the 39 countries, except in Model 2, which is based on 28 countries because of a missing value in Tourism in one of the countries (Taiwan).

\begin{tabular}{|c|c|c|c|c|c|c|c|c|c|}
\hline \multirow[b]{2}{*}{ Predictor } & \multicolumn{3}{|c|}{ Model1 } & \multicolumn{3}{|c|}{ Model 2} & \multicolumn{3}{|c|}{ Model 3} \\
\hline & b & $t$ & $p$ & b & $t$ & $\mathrm{p}$ & b & $t$ & $\mathrm{p}$ \\
\hline Intercept & 7.008 & 28.009 & $<.001$ & 6.980 & 31.961 & $<.001$ & 7.082 & 42.099 & $<.001$ \\
\hline Day & 0.124 & 8.312 & 0.001 & 0.121 & 11.833 & $<.001$ & 0.125 & 15.176 & $<.001$ \\
\hline $\begin{array}{l}\text { Relational } \\
\text { Mobility }\end{array}$ & 1.956 & 2.795 & 0.010 & 1.254 & 1.655 & 0.116 & 1.302 & 1.910 & 0.071 \\
\hline Population & 0.768 & 3.670 & 0.001 & 0.445 & 1.596 & 0.127 & 0.435 & 1.670 & 0.110 \\
\hline Migration & & & & 0.364 & 0.791 & 0.439 & & & \\
\hline GDP & & & & 0.212 & 0.676 & 0.509 & 0.220 & 1.165 & 0.257 \\
\hline $\begin{array}{l}\text { Population } \\
\text { Density }\end{array}$ & & & & -0.352 & -1.591 & 0.128 & -0.380 & -2.018 & 0.056 \\
\hline Tourism & & & & 0.262 & 1.499 & 0.150 & 0.242 & 1.545 & 0.137 \\
\hline $\begin{array}{l}\text { Percent } \\
\text { Urban }\end{array}$ & & & & -0.114 & -0.494 & 0.628 & & & \\
\hline Median Age & & & & -0.041 & -0.197 & 0.846 & & & \\
\hline $\begin{array}{l}\text { Day x } \\
\text { Relational } \\
\text { Mobility }\end{array}$ & 0.126 & 3.425 & 0.002 & 0.079 & 2.425 & 0.027 & 0.085 & 2.651 & 0.015 \\
\hline $\begin{array}{l}\text { Day } \mathrm{x} \\
\text { Population }\end{array}$ & 0.046 & 4.202 & $<.001$ & 0.031 & 2.583 & 0.019 & 0.031 & 2.484 & 0.022 \\
\hline $\begin{array}{l}\text { Day } \mathrm{x} \\
\text { Migration }\end{array}$ & & & & 0.014 & 0.682 & 0.504 & & & \\
\hline Day x GDP & & & & 0.031 & 2.226 & 0.039 & 0.019 & 2.111 & 0.047 \\
\hline $\begin{array}{l}\text { Day x } \\
\text { Population } \\
\text { Density }\end{array}$ & & & & -0.019 & -1.925 & 0.068 & -0.016 & -1.761 & 0.090 \\
\hline $\begin{array}{l}\text { Day } \mathrm{x} \\
\text { Tourism }\end{array}$ & & & & 0.016 & 2.090 & 0.048 & 0.017 & 2.158 & 0.041 \\
\hline $\begin{array}{l}\text { Day x Percent } \\
\text { Urban }\end{array}$ & & & & -0.014 & -1.407 & 0.178 & & & \\
\hline $\begin{array}{l}\text { Day x Median } \\
\text { Age }\end{array}$ & & & & -0.010 & -1.128 & 0.276 & & & \\
\hline $\begin{array}{l}R^{2} \text { fixed } \\
\text { effects }\end{array}$ & & 0.279 & & & 0.310 & & & 0.312 & \\
\hline $\begin{array}{l}\mathrm{R}^{2} \text { fixed and } \\
\text { random } \\
\text { effects }\end{array}$ & & 0.377 & & & 0.381 & & & 0.376 & \\
\hline
\end{tabular}


Table S7-B.

Regression coefficients predicting the number of confirmed cases, with testing availability (referred to as Testing) used as a covariate. A. Model 1 includes total population (called Population) and Testing. Model 2 includes all demographic covariates and Testing. Model 3 includes only those demographic variables that prove at least marginally significant in Model 2 and Testing. The results are based on 29 of the 39 countries, except in Model 2, which is based on 28 countries because of a missing value in Tourism in one of the countries (Taiwan).

\begin{tabular}{|c|c|c|c|c|c|c|c|c|c|}
\hline \multirow[b]{2}{*}{ Predictor } & \multicolumn{3}{|c|}{ Model 1} & \multicolumn{3}{|c|}{ Model 2} & \multicolumn{3}{|c|}{ Model 3} \\
\hline & $b$ & $t$ & $p$ & b & $\mathrm{t}$ & $\mathrm{p}$ & $b$ & $\mathrm{t}$ & $\mathrm{p}$ \\
\hline Intercept & 7.000 & 26.609 & $<.001$ & 7.012 & 36.280 & $<.001$ & 7.114 & 48.948 & $<.001$ \\
\hline Day & 0.124 & 7.977 & 0.001 & 0.120 & 14.178 & $<.001$ & 0.125 & 18.349 & $<.001$ \\
\hline $\begin{array}{l}\text { Relational } \\
\text { Mobility }\end{array}$ & 1.901 & 2.626 & 0.015 & 1.242 & 1.634 & 0.120 & 1.228 & 1.806 & 0.085 \\
\hline Testing & 0.040 & 0.255 & 0.801 & -0.119 & -0.726 & 0.477 & -0.112 & -0.734 & 0.471 \\
\hline Population & 0.790 & 3.425 & 0.002 & 0.321 & 1.118 & 0.278 & 0.317 & 1.206 & 0.241 \\
\hline Migration & & & & 0.382 & 0.810 & 0.429 & & & \\
\hline GDP & & & & 0.261 & 0.815 & 0.426 & 0.268 & 1.347 & 0.192 \\
\hline $\begin{array}{l}\text { Population } \\
\text { Density }\end{array}$ & & & & -0.436 & -1.782 & 0.092 & -0.469 & -2.277 & 0.033 \\
\hline Tourism & & & & 0.275 & 1.525 & 0.145 & 0.256 & 1.593 & 0.126 \\
\hline Percent Urban & & & & -0.166 & -0.720 & 0.481 & & & \\
\hline Median Age & & & & -0.007 & -0.036 & 0.971 & & & \\
\hline $\begin{array}{l}\text { Day } x \text { Relational } \\
\text { Mobility }\end{array}$ & 0.125 & 3.237 & 0.003 & 0.077 & 2.310 & 0.033 & 0.076 & 2.388 & 0.026 \\
\hline Day x Testing & -0.004 & -0.537 & 0.596 & -0.011 & -1.497 & 0.152 & -0.009 & -1.310 & 0.204 \\
\hline Day $x$ Population & 0.044 & 3.583 & 0.002 & 0.023 & 1.832 & 0.083 & 0.024 & 1.920 & 0.069 \\
\hline Day $x$ Migration & & & & 0.018 & 0.875 & 0.393 & & & \\
\hline Day x GDP & & & & 0.033 & 2.327 & 0.032 & 0.024 & 2.526 & 0.020 \\
\hline $\begin{array}{l}\text { Day x Population } \\
\text { Density }\end{array}$ & & & & -0.025 & -2.372 & 0.029 & -0.024 & -2.433 & 0.024 \\
\hline Day x Tourism & & & & 0.016 & 1.986 & 0.062 & 0.015 & 2.050 & 0.053 \\
\hline $\begin{array}{l}\text { Day } x \text { Percent } \\
\text { Urban }\end{array}$ & & & & -0.016 & -1.567 & 0.135 & & & \\
\hline $\begin{array}{l}\text { Day } x \text { Median } \\
\text { Age }\end{array}$ & & & & -0.008 & -0.830 & 0.417 & & & \\
\hline$R^{2}$ fixed effects & & 0.698 & & & 0.787 & & & 0.797 & \\
\hline$R^{2}$ fixed and & & 0.968 & & & 0.967 & & & 0.966 & \\
\hline
\end{tabular}


Table S8.

Regression coefficients predicting the number of confirmed cases over the first 30 days of country-wise outbreaks. Day 1 is defined as the date when 20 cases (rather than 100 cases as in the main analysis) were confirmed in each country. The Models vary in the covariates included. Model 1 includes only total population (called Population). Model 2 includes all demographic covariates. Model 3 includes only those that prove at least marginally significant in Model 2. The results are based on 39 countries, except in Model 2, which is based on 38 countries because of a missing value in Tourism in one of the countries (Taiwan).

\begin{tabular}{|c|c|c|c|c|c|c|c|c|c|}
\hline \multirow[b]{2}{*}{ Predictor } & \multicolumn{3}{|c|}{ Model 1} & \multicolumn{3}{|c|}{ Model 2} & \multicolumn{3}{|c|}{ Model 3} \\
\hline & $b$ & $\mathrm{t}$ & $p$ & $b$ & $\mathrm{t}$ & $p$ & $\mathrm{~b}$ & $\mathrm{t}$ & $p$ \\
\hline Intercept & 5.900 & $\begin{array}{r}16.75 \\
6\end{array}$ & $<.001$ & 5.785 & 23.964 & $<.001$ & 5.805 & 25.019 & $<.001$ \\
\hline Day & 0.157 & 7.883 & 0.005 & 0.154 & 16.072 & 0.045 & 0.152 & 17.168 & 0.058 \\
\hline $\begin{array}{l}\text { Relational } \\
\text { Mobility }\end{array}$ & 3.216 & 4.138 & $<.001$ & 2.724 & 3.246 & 0.003 & 2.605 & 3.494 & 0.002 \\
\hline Population & 0.458 & 3.112 & 0.004 & 0.155 & 0.923 & 0.364 & 0.181 & 1.194 & 0.243 \\
\hline Migration & & & & 0.245 & 1.711 & 0.098 & 0.232 & 1.789 & 0.083 \\
\hline GDP & & & & -0.139 & -0.504 & 0.618 & & & \\
\hline $\begin{array}{l}\text { Population } \\
\text { Density }\end{array}$ & & & & -0.116 & -0.606 & 0.549 & -0.196 & -1.464 & 0.153 \\
\hline Tourism & & & & 0.366 & 2.191 & 0.037 & 0.340 & 2.203 & 0.035 \\
\hline Percent Urban & & & & -0.020 & -0.124 & 0.902 & & & \\
\hline Median Age & & & & -0.010 & -0.049 & 0.961 & & & \\
\hline $\begin{array}{l}\text { Day x } \\
\text { Relational } \\
\text { Mobility }\end{array}$ & 0.182 & 4.061 & $<.001$ & 0.126 & 2.900 & 0.016 & 0.131 & 3.350 & 0.010 \\
\hline $\begin{array}{l}\text { Day x } \\
\text { Population }\end{array}$ & 0.039 & 4.559 & $<.001$ & 0.024 & 2.691 & 0.013 & 0.021 & 2.547 & 0.018 \\
\hline Day x Migration & & & & 0.014 & 1.794 & 0.084 & 0.015 & 1.953 & 0.060 \\
\hline Day x GDP & & & & -0.000 & -0.029 & 0.977 & & & \\
\hline $\begin{array}{l}\text { Day x } \\
\text { Population } \\
\text { Density }\end{array}$ & & & & -0.019 & -1.792 & 0.084 & -0.015 & -2.003 & 0.055 \\
\hline Day $x$ Tourism & & & & 0.017 & 1.856 & 0.074 & 0.022 & 2.558 & 0.019 \\
\hline $\begin{array}{l}\text { Day x Percent } \\
\text { Urban }\end{array}$ & & & & -0.001 & -0.067 & 0.947 & & & \\
\hline $\begin{array}{l}\text { Day x Median } \\
\text { Age }\end{array}$ & & & & 0.017 & 1.517 & 0.150 & & & \\
\hline$R^{2}$ fixed effects & & 0.648 & & & 0.724 & & & 0.732 & \\
\hline $\begin{array}{l}\mathrm{R}^{2} \text { fixed and } \\
\text { random effects }\end{array}$ & & 0.974 & & & 0.970 & & & 0.969 & \\
\hline
\end{tabular}


Table S9.

Regression coefficients predicting the number of confirmed cases $(A)$ and deaths $(B)$ over the first 15 (rather than 30 as in the main analysis) days of country-wise outbreaks. Models vary in the covariates included. Model 1 includes only total population (called Population). Model 2 includes all demographic covariates. Model 3 includes only those that prove at least marginally significant in Model 2. The results are based on 39 countries, except in Model 2, which is based on 38 countries because of a missing value in Tourism in one of the countries (Taiwan).

Model 1

Model 2

Model 3

\begin{tabular}{lrrrrrrrrr} 
A. Predictor & $\mathrm{b}$ & $\mathrm{t}$ & $\mathrm{p}$ & $\mathrm{b}$ & $\mathrm{t}$ & $\mathrm{p}$ & $\mathrm{b}$ & $\mathrm{t}$ & $\mathrm{p}$ \\
\hline Intercept & 7.338 & 20.427 & $<.001$ & 7.315 & 50.408 & $<.001$ & 7.285 & 42.626 & $<.001$ \\
Day & 0.174 & 7.277 & 0.004 & 0.169 & 19.411 & $<.001$ & 0.169 & 18.495 & 0.022 \\
Relational & 2.763 & 3.035 & 0.005 & 1.378 & 1.679 & 0.104 & 1.745 & 2.182 & 0.044 \\
Mobility & & & & & & & & & \\
Population & 0.652 & 3.871 & 0.001 & 0.309 & 1.728 & 0.095 & & & \\
Migration & & & & 0.338 & 2.042 & 0.050 & 0.339 & 2.202 & 0.035 \\
GDP & & & & 0.076 & 0.250 & 0.805 & & & \\
Population & & & & -0.549 & -2.531 & 0.017 & -0.475 & -3.109 & 0.004 \\
Density & & & & & & & & & \\
Tourism & & & & 0.379 & 2.017 & 0.053 & 0.579 & 3.687 & 0.001 \\
Percent Urban & & & & 0.070 & 0.381 & 0.706 & & & \\
Median Age & & & & 0.199 & 0.939 & 0.356 & & & \\
Day x & 0.198 & 3.465 & 0.001 & 0.092 & 1.872 & 0.071 & 0.118 & 2.531 & 0.029 \\
Relational & & & & & & & & & \\
Mobility & & & & & & & & & \\
Day x & 0.042 & 3.948 & 0.001 & 0.018 & 1.693 & 0.101 & & & \\
Population & & & & & & & & & \\
Day x & & & & 0.021 & 2.100 & 0.045 & 0.023 & 2.522 & 0.017 \\
Migration & & & & & & & & & \\
Day x GDP & & & & 0.013 & 0.717 & 0.479 & & & \\
Day x & & & & -0.036 & -2.778 & 0.009 & -0.028 & -3.023 & 0.005 \\
Population & & & & & & & & & \\
Density & & & & 0.027 & 2.359 & 0.025 & 0.041 & 4.358 & $<.001$ \\
Day x Tourism & & & & 0.004 & 0.338 & 0.738 & & & \\
Day x Percent & & & & & & & & & \\
Urban & & & 0.007 & 0.547 & 0.588 & & & \\
Day x Median & & & & & 0.733 & & & 0.448 & \\
Age & & & & & 0.985 & & & 0.990 & \\
$R^{2}$ fixed effects & 0.319 & & & & & & & \\
$\mathrm{R}^{2}$ fixed and \\
random effects
\end{tabular}




\begin{tabular}{|c|c|c|c|c|c|c|c|c|c|}
\hline \multirow[b]{2}{*}{ B. Predictor } & \multicolumn{3}{|c|}{ Model 1} & \multicolumn{3}{|c|}{ Model 2} & \multicolumn{3}{|c|}{ Model 3} \\
\hline & b & $t$ & $\mathrm{p}$ & $b$ & $\mathrm{t}$ & $\mathrm{p}$ & $\mathrm{b}$ & $t$ & $\mathrm{p}$ \\
\hline Intercept & 2.918 & 6.481 & 0.003 & 2.960 & 6.396 & 0.016 & 2.855 & 6.731 & 0.002 \\
\hline Day & 0.187 & 6.142 & 0.003 & 0.190 & 6.485 & 0.010 & 0.183 & 6.329 & 0.002 \\
\hline $\begin{array}{l}\text { Relational } \\
\text { Mobility }\end{array}$ & 2.123 & 1.434 & 0.163 & 0.755 & 0.429 & 0.672 & 2.127 & 1.402 & 0.172 \\
\hline Population & 0.435 & 1.604 & 0.119 & 0.308 & 0.881 & 0.386 & & & \\
\hline Migration & & & & 0.235 & 0.782 & 0.441 & & & \\
\hline GDP & & & & 0.509 & 0.879 & 0.387 & & & \\
\hline $\begin{array}{l}\text { Population } \\
\text { Density }\end{array}$ & & & & -0.623 & -1.551 & 0.132 & -0.263 & -0.940 & 0.354 \\
\hline Tourism & & & & 0.054 & 0.153 & 0.879 & & & \\
\hline Percent Urban & & & & 0.100 & 0.296 & 0.770 & & & \\
\hline Median Age & & & & 0.074 & 0.168 & 0.868 & & & \\
\hline $\begin{array}{l}\text { Day x } \\
\text { Relational } \\
\text { Mobility }\end{array}$ & 0.140 & 1.483 & 0.148 & 0.046 & 0.421 & 0.678 & 0.134 & 1.399 & 0.172 \\
\hline $\begin{array}{l}\text { Day } \mathrm{x} \\
\text { Population }\end{array}$ & 0.026 & 1.485 & 0.147 & 0.020 & 0.940 & 0.356 & & & \\
\hline $\begin{array}{l}\text { Day } \mathrm{x} \\
\text { Migration }\end{array}$ & & & & 0.014 & 0.738 & 0.467 & & & \\
\hline Day x GDP & & & & 0.041 & 1.144 & 0.262 & & & \\
\hline $\begin{array}{l}\text { Day } x \\
\text { Population } \\
\text { Density }\end{array}$ & & & & -0.046 & -1.854 & 0.074 & -0.019 & -1.082 & 0.287 \\
\hline Day $\times$ Tourism & & & & 0.000 & 0.001 & 0.999 & & & \\
\hline $\begin{array}{l}\text { Day x Percent } \\
\text { Urban }\end{array}$ & & & & 0.001 & 0.031 & 0.975 & & & \\
\hline $\begin{array}{l}\text { Day x Median } \\
\text { Age }\end{array}$ & & & & 0.009 & 0.347 & 0.731 & & & \\
\hline$R^{2}$ fixed effects & & 0.165 & & & 0.181 & & & 0.153 & \\
\hline $\begin{array}{l}\mathrm{R}^{2} \text { fixed and } \\
\text { random effects }\end{array}$ & & 0.984 & & & 0.985 & & & 0.984 & \\
\hline
\end{tabular}


Table S10.

Regression coefficients predicting the number of confirmed cases $(A)$ and deaths $(B)$ over the first 60 (rather than 30 as in the main analysis) days of country-wise outbreaks. Models vary in the covariates included. Model 1 includes only total population (called Population). Model 2 includes all demographic covariates. Model 3 includes only those that prove at least marginally significant in Model 2. The results are based on 39 countries, except in Model 2, which is based on 38 countries because of a missing value in Tourism in one of the countries (Taiwan).

\begin{tabular}{|c|c|c|c|c|c|c|c|c|c|}
\hline \multirow[b]{2}{*}{ A. Predictor } & \multicolumn{3}{|c|}{ Model 1} & \multicolumn{4}{|c|}{ Model 2} & \multicolumn{2}{|c|}{ Model 3} \\
\hline & $\mathrm{b}$ & $\mathrm{t}$ & $\mathrm{p}$ & $\mathrm{b}$ & $\mathrm{t}$ & $\mathrm{p}$ & b & $\mathrm{t}$ & $\mathrm{p}$ \\
\hline Intercept & 8.133 & 15.441 & 0.001 & 7.925 & 37.700 & 0.023 & 7.987 & 22.898 & 0.001 \\
\hline Day & 0.071 & 7.174 & 0.010 & 0.068 & 12.734 & 0.172 & 0.067 & 18.581 & 0.012 \\
\hline $\begin{array}{l}\text { Relational } \\
\text { Mobility }\end{array}$ & 2.925 & 3.430 & 0.002 & 1.904 & 2.273 & 0.038 & 2.571 & 3.134 & 0.004 \\
\hline Population & 1.054 & 6.152 & $<.001$ & 0.649 & 3.848 & 0.001 & 0.770 & 4.516 & $<.001$ \\
\hline Migration & & & & 0.333 & 2.259 & 0.033 & & & \\
\hline GDP & & & & 0.244 & 0.867 & 0.393 & & & \\
\hline $\begin{array}{l}\text { Population } \\
\text { Density }\end{array}$ & & & & -0.313 & -1.597 & 0.122 & & & \\
\hline Tourism & & & & 0.446 & 2.605 & 0.014 & 0.409 & 2.449 & 0.020 \\
\hline Percent Urban & & & & -0.002 & -0.015 & 0.988 & & & \\
\hline Median Age & & & & 0.125 & 0.593 & 0.560 & & & \\
\hline $\begin{array}{l}\text { Day x } \\
\text { Relational } \\
\text { Mobility }\end{array}$ & 0.048 & 2.261 & 0.030 & 0.037 & 1.969 & 0.067 & 0.039 & 2.437 & 0.038 \\
\hline $\begin{array}{l}\text { Day } \mathrm{x} \\
\text { Population }\end{array}$ & 0.023 & 5.777 & $<.001$ & 0.019 & 5.031 & $<.001$ & 0.014 & 4.134 & $<.001$ \\
\hline Day $\times$ Migration & & & & 0.005 & 1.650 & 0.115 & & & \\
\hline Day x GDP & & & & 0.009 & 1.503 & 0.144 & & & \\
\hline $\begin{array}{l}\text { Day } x \\
\text { Population } \\
\text { Density }\end{array}$ & & & & 0.001 & 0.349 & 0.731 & & & \\
\hline Day $x$ Tourism & & & & 0.009 & 2.524 & 0.018 & 0.016 & 4.312 & $<.001$ \\
\hline $\begin{array}{l}\text { Day x Percent } \\
\text { Urban }\end{array}$ & & & & -0.002 & -0.627 & 0.537 & & & \\
\hline $\begin{array}{l}\text { Day x Median } \\
\text { Age }\end{array}$ & & & & 0.001 & 0.131 & 0.897 & & & \\
\hline$R^{2}$ fixed effects & & 0.561 & & & 0.752 & & & 0.666 & \\
\hline $\begin{array}{l}\mathrm{R}^{2} \text { fixed and } \\
\text { random effects }\end{array}$ & & 0.952 & & & 0.938 & & & 0.938 & \\
\hline
\end{tabular}




\begin{tabular}{|c|c|c|c|c|c|c|c|c|c|}
\hline \multirow[b]{2}{*}{ B. Predictor } & \multicolumn{2}{|c|}{ Model 1} & \multicolumn{4}{|c|}{ Model 2} & \multicolumn{3}{|c|}{ Model 3} \\
\hline & $b$ & $\mathrm{t}$ & $\mathrm{p}$ & $b$ & $\mathrm{t}$ & $\mathrm{p}$ & $b$ & $t$ & $\mathrm{p}$ \\
\hline Intercept & 4.045 & 7.181 & 0.004 & 3.982 & 9.089 & 0.009 & 3.939 & 9.486 & 0.001 \\
\hline Day & 0.098 & 5.515 & 0.009 & 0.088 & 12.220 & 0.005 & 0.090 & 9.412 & 0.006 \\
\hline $\begin{array}{l}\text { Relational } \\
\text { Mobility }\end{array}$ & 3.061 & 2.431 & 0.020 & 1.849 & 1.400 & 0.173 & 3.039 & 2.536 & 0.016 \\
\hline Population & 0.956 & 4.027 & $<.001$ & 0.704 & 2.671 & 0.014 & 0.657 & 2.722 & 0.011 \\
\hline Migration & & & & 0.337 & 1.578 & 0.127 & 0.349 & 1.676 & 0.104 \\
\hline GDP & & & & 0.497 & 1.186 & 0.245 & & & \\
\hline $\begin{array}{l}\text { Population } \\
\text { Density }\end{array}$ & & & & -0.524 & -1.827 & 0.079 & & & \\
\hline Tourism & & & & 0.341 & 1.348 & 0.188 & 0.447 & 1.814 & 0.079 \\
\hline Percent Urban & & & & 0.031 & 0.130 & 0.898 & & & \\
\hline Median Age & & & & 0.226 & 0.698 & 0.491 & & & \\
\hline $\begin{array}{l}\text { Day x } \\
\text { Relational } \\
\text { Mobility }\end{array}$ & 0.087 & 3.543 & 0.001 & 0.073 & 2.955 & 0.007 & 0.086 & 3.719 & 0.001 \\
\hline $\begin{array}{l}\text { Day } \mathrm{x} \\
\text { Population }\end{array}$ & 0.032 & 6.388 & $<.001$ & 0.021 & 4.271 & $<.001$ & 0.020 & 4.251 & $<.001$ \\
\hline Day $x$ Migration & & & & 0.007 & 1.731 & 0.095 & 0.007 & 1.712 & 0.097 \\
\hline Day x GDP & & & & 0.006 & 0.809 & 0.425 & & & \\
\hline $\begin{array}{l}\text { Day x } \\
\text { Population } \\
\text { Density }\end{array}$ & & & & -0.007 & -1.337 & 0.192 & & & \\
\hline Day x Tourism & & & & 0.015 & 3.045 & 0.005 & 0.015 & 3.203 & 0.003 \\
\hline $\begin{array}{l}\text { Day x Percent } \\
\text { Urban }\end{array}$ & & & & -0.005 & -1.167 & 0.253 & & & \\
\hline $\begin{array}{l}\text { Day x Median } \\
\text { Age }\end{array}$ & & & & 0.009 & 1.435 & 0.162 & & & \\
\hline$R^{2}$ fixed effects & & 0.540 & & & 0.652 & & & 0.620 & \\
\hline $\begin{array}{l}\mathrm{R}^{2} \text { fixed and } \\
\text { random effects }\end{array}$ & & 0.948 & & & 0.940 & & & 0.936 & \\
\hline
\end{tabular}


Table S11-A.

Regression coefficients predicting the number of confirmed cases for the 39 countries with RM scores and an additional 46 countries with interpolated scores. Models vary in the covariates included. Model 1 includes only total population (called Population). Model 2 includes all demographic covariates. Model 3 includes only those that prove at least marginally significant in Model 2. The results are based on 83 countries in Model 1 due to missing COVID-19 data for a country and a missing value for population size for another. Model 2 included 78 countries due to missing data on a few demographic variables. Model 3 included 82 countries.

\begin{tabular}{|c|c|c|c|c|c|c|c|c|c|}
\hline \multirow[b]{2}{*}{ Predictor } & \multicolumn{3}{|c|}{ Model 1} & \multicolumn{3}{|c|}{ Model 2} & \multicolumn{3}{|c|}{ Model 3} \\
\hline & $b$ & $\mathrm{t}$ & $p$ & $\mathrm{~b}$ & $\mathrm{p}$ & & b & $\mathrm{t}$ & $\mathrm{p}$ \\
\hline Intercept & 6.721 & 30.909 & $<.001$ & 6.741 & 43.771 & $<.001$ & 6.706 & 51.693 & $<.001$ \\
\hline Day & 0.111 & 7.779 & 0.002 & 0.110 & 11.604 & 0.001 & 0.107 & 14.956 & 0.001 \\
\hline $\begin{array}{l}\text { Relational } \\
\text { Mobility }\end{array}$ & 2.686 & 3.433 & 0.001 & 1.471 & 2.368 & 0.021 & 1.634 & 2.497 & 0.016 \\
\hline Population & 0.140 & 1.433 & 0.156 & 0.028 & 0.369 & 0.713 & & & \\
\hline Migration & & & & 0.183 & 1.873 & 0.065 & 0.131 & 1.535 & 0.129 \\
\hline GDP & & & & -0.052 & -0.424 & 0.673 & & & \\
\hline $\begin{array}{l}\text { Population } \\
\text { Density }\end{array}$ & & & & -0.260 & -3.064 & 0.003 & & & \\
\hline Tourism & & & & 0.489 & 6.040 & $<.001$ & 0.530 & 5.952 & $<.001$ \\
\hline Percent Urban & & & & 0.198 & 1.639 & 0.107 & & & \\
\hline Median Age & & & & -0.089 & -0.736 & 0.465 & & & \\
\hline $\begin{array}{l}\text { Day x } \\
\text { Relational } \\
\text { Mobility }\end{array}$ & 0.148 & 3.623 & 0.001 & 0.095 & 2.856 & 0.006 & 0.090 & 2.793 & 0.007 \\
\hline $\begin{array}{l}\text { Day } \mathrm{x} \\
\text { Population }\end{array}$ & 0.007 & 1.495 & 0.139 & 0.002 & 0.459 & 0.648 & & & \\
\hline $\begin{array}{l}\text { Day } \mathrm{x} \\
\text { Migration }\end{array}$ & & & & 0.011 & 2.045 & 0.045 & 0.008 & 1.994 & 0.050 \\
\hline Day x GDP & & & & -0.002 & -0.307 & 0.760 & & & \\
\hline $\begin{array}{l}\text { Day x } \\
\text { Population } \\
\text { Density }\end{array}$ & & & & -0.006 & -1.231 & 0.223 & & & \\
\hline Day x Tourism & & & & 0.029 & 6.692 & $<.001$ & 0.030 & 6.967 & $<.001$ \\
\hline $\begin{array}{l}\text { Day x Percent } \\
\text { Urban }\end{array}$ & & & & 0.008 & 1.247 & 0.217 & & & \\
\hline $\begin{array}{l}\text { Day x Median } \\
\text { Age }\end{array}$ & & & & -0.009 & -1.361 & 0.178 & & & \\
\hline$R^{2}$ fixed effects & & 0.472 & & & 0.696 & & & 0.645 & \\
\hline $\begin{array}{l}\mathrm{R}^{2} \text { fixed and } \\
\text { random effects }\end{array}$ & & 0.972 & & & 0.970 & & & 0.969 & \\
\hline
\end{tabular}


Table S11-B.

Regression coefficients predicting the number of confirmed deaths for the 39 countries with RM scores and an additional 46 countries with interpolated scores. Models vary in the covariates included. Model 1 includes only total population (called Population). Model 2 includes all demographic covariates. Model 3 includes only those that prove at least marginally significant in Model 2. The results are based on 82 countries in Model 1, due to missing COVID-19 data for two countries and a missing value for population size in one. Model 2 included 77 countries due to missing data on a few demographic variables. Model 3 included 81 countries.

\begin{tabular}{|c|c|c|c|c|c|c|c|c|c|}
\hline \multirow[b]{2}{*}{ Predictor } & \multicolumn{3}{|c|}{ Model 1} & \multicolumn{3}{|c|}{ Model 2} & \multicolumn{3}{|c|}{ Model 3} \\
\hline & b & $\mathrm{t}$ & $\mathrm{p}$ & $\mathrm{b}$ & $\mathrm{t}$ & & $b$ & $\mathrm{t}$ & $\mathrm{p}$ \\
\hline Intercept & 2.370 & 11.322 & $<.001$ & 2.432 & 14.744 & 0.001 & 2.405 & 14.423 & $<.001$ \\
\hline Day & 0.131 & 9.307 & $<.001$ & 0.136 & 12.366 & $<.001$ & 0.132 & 12.309 & $<.001$ \\
\hline $\begin{array}{l}\text { Relational } \\
\text { Mobility }\end{array}$ & 3.261 & 3.257 & 0.002 & 1.942 & 1.957 & 0.059 & 1.816 & 1.877 & 0.067 \\
\hline Population & 0.144 & 1.084 & 0.282 & 0.016 & 0.117 & 0.907 & & & \\
\hline Migration & & & & 0.268 & 1.580 & 0.119 & 0.262 & 1.994 & 0.050 \\
\hline GDP & & & & -0.131 & -0.627 & 0.533 & & & \\
\hline $\begin{array}{l}\text { Population } \\
\text { Density }\end{array}$ & & & & -0.242 & -1.657 & 0.102 & -0.258 & -1.945 & 0.056 \\
\hline Tourism & & & & 0.375 & 2.673 & 0.009 & 0.444 & 3.394 & 0.001 \\
\hline Percent Urban & & & & 0.130 & 0.671 & 0.508 & & & \\
\hline Median Age & & & & 0.165 & 0.873 & 0.394 & & & \\
\hline $\begin{array}{l}\text { Day x } \\
\text { Relational } \\
\text { Mobility }\end{array}$ & 0.202 & 3.831 & $<.001$ & 0.122 & 2.538 & 0.014 & 0.113 & 2.341 & 0.022 \\
\hline $\begin{array}{l}\text { Day } \mathrm{x} \\
\text { Population }\end{array}$ & 0.009 & 1.362 & 0.177 & 0.002 & 0.295 & 0.769 & & & \\
\hline $\begin{array}{l}\text { Day } \mathrm{x} \\
\text { Migration }\end{array}$ & & & & 0.014 & 1.836 & 0.071 & 0.014 & 2.230 & 0.029 \\
\hline Day x GDP & & & & -0.005 & -0.499 & 0.619 & & & \\
\hline $\begin{array}{l}\text { Day x } \\
\text { Population } \\
\text { Density }\end{array}$ & & & & -0.012 & -1.774 & 0.081 & -0.012 & -1.962 & 0.053 \\
\hline Day x Tourism & & & & 0.026 & 4.054 & $<.001$ & 0.031 & 4.872 & $<.001$ \\
\hline $\begin{array}{l}\text { Day } \mathrm{x} \text { Percent } \\
\text { Urban }\end{array}$ & & & & 0.005 & 0.544 & 0.589 & & & \\
\hline $\begin{array}{l}\text { Day } x \text { Median } \\
\text { Age }\end{array}$ & & & & 0.011 & 1.153 & 0.255 & & & \\
\hline$R^{2}$ fixed effects & & 0.449 & & & 0.578 & & & 0.547 & \\
\hline $\begin{array}{l}R^{2} \text { fixed and } \\
\text { random effects }\end{array}$ & & 0.966 & & & 0.967 & & & 0.966 & \\
\hline
\end{tabular}


Figure S1.

A correlation matrix of the demographic and cultural variables included in the present analysis. Only countries with RM scores are included to compute each correlation. Some covariates, particularly, cultural covariates, were not available for some countries. Each coefficient is based on the maximal number of countries that is possible given the data.

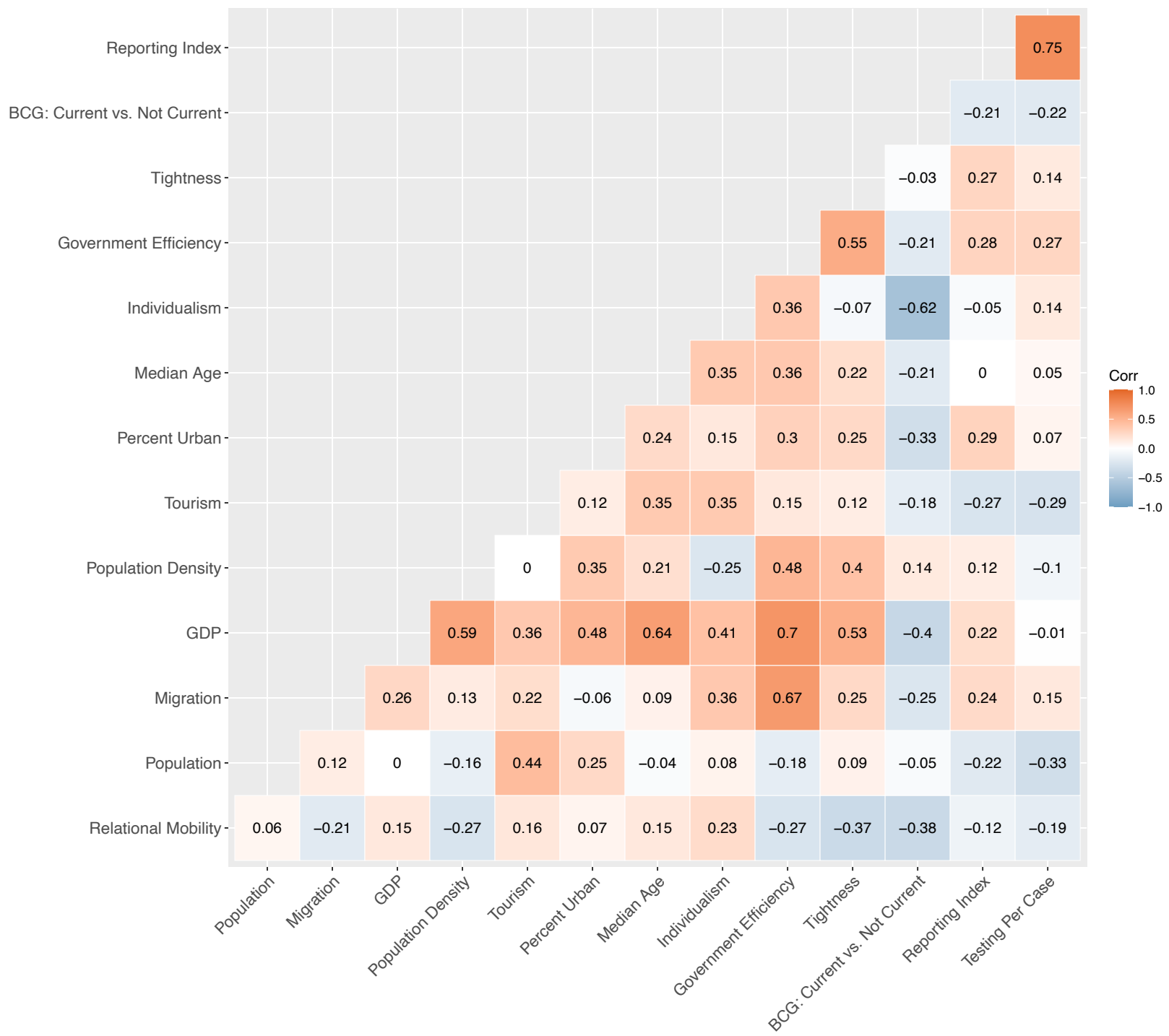


Figure S2.

The rate of growth of confirmed cases and deaths of COVID-19 during the first 30 days of country-wise outbreaks after interpolation. A. Country-wise growth rates of confirmed cases as a function of RM for 83 countries. B. Country-wise growth rates of deaths as a function of RM for 82 countries.
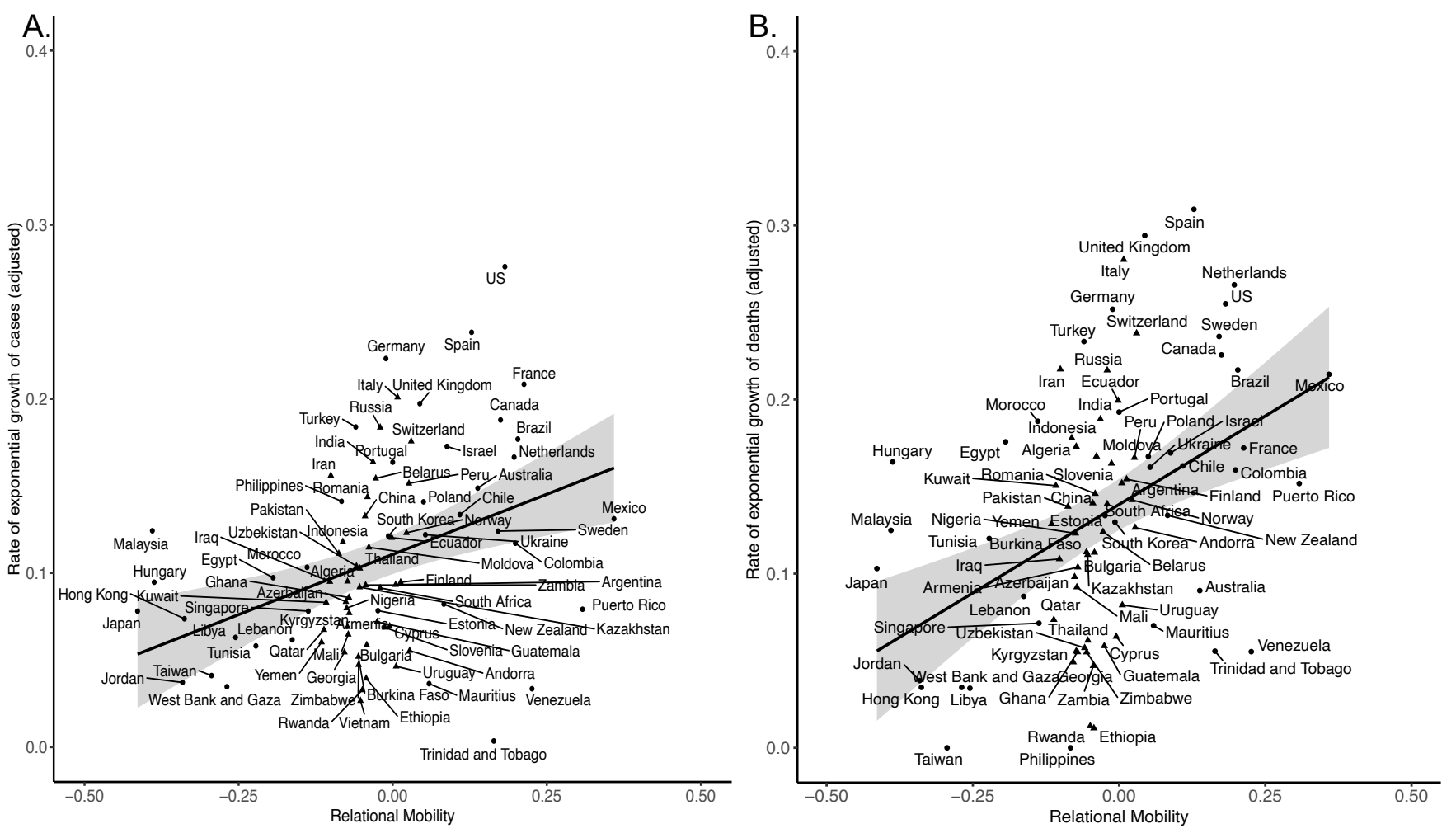

nterpolated 